\title{
Effect of N Fertilization on Pod and Seed Characteristics of Okra in Relation to Plant Part
}

\author{
Mohammadi Ghadir ${ }^{1}$, Khah E. M. ${ }^{1}$, Spyridon A. Petropoulos ${ }^{2}$, Chachalis D. ${ }^{3}$, Garip Yarsi ${ }^{4}$, \\ \& Muhammad A. Anjum ${ }^{5}$ \\ ${ }^{1}$ Laboratory of Genetics and Plant Breeding, Department of Agriculture, Crop Production and Rural \\ Environment, School of Agricultural Sciences, University of Thessaly, Nea Ionia, Volos, Greece \\ ${ }^{2}$ Laboratory of Vegetable Production, University of Thessaly, Nea Ionia, Volos, Greece \\ ${ }^{3}$ Benaki Phytopathological Institute, Kifissia, Athens, Greece \\ ${ }^{4}$ Adiyaman University Plant and Animal Production, Adiyaman, Turkey \\ ${ }^{5}$ Department of Horticulture, Faculty of Agricultural Sciences \& Technology, Bahauddin Zakariya University, \\ Multan, Pakistan \\ Correspondence: Spyridon A. Petropoulos, Laboratory of Vegetable Production, University of Thessaly, Fytokou \\ Str. Nea Ionia, Volos 38446, Greece. Tel: 30-242-109-3196. Fax: 30-242-109-3144. E-mail: \\ fangio57gr@gmail.com
}

Received: July 25, 2014 Accepted: August 15, 2014 Online Published: December 15, 2014

doi:10.5539/jas.v7n1p54 URL: http://dx.doi.org/10.5539/jas.v7n1p54

\begin{abstract}
Four cultivars of okra (Abelmoschus esculentus [L.] Moench. cv. 'Boyiatiou', 'Veloudo', 'Pylaias' and 'Clemson') were sown on $1^{\text {st }}$ of May (Experiment 1) and $20^{\text {th }}$ of May, 2011 (Experiment 2) and fertilized with three nitrogen levels $\left(150,300\right.$ and $\left.450 \mathrm{mg} \mathrm{N}^{-1}\right)$. The pods were harvested from three plant parts (upper, middle and lower part) and the seeds were extracted from the pods either immediately after harvest or after storage for 15 days at room temperature. The present study provides new information on the seed quality of four cultivars of okra in relation to $\mathrm{N}$ level and the region (upper, lower and middle) of the plant from which harvest took place. $\mathrm{N}$ application generally increased flowering, but pod set increased only in 'Boyiatiou'. Pod dimensions (length and diameter) were largely unaffected by $\mathrm{N}$ level, irrespective of the position of the pods on the plant, as was the 100 -seed weight and seed germination. The slight (insignificant) increase in seed germination of 'Boyiatiou' in response to $\mathrm{N}$ is worthy of further study in view of the susceptibility of this cultivar to seed hardness. There was no benefit of pod after-ripening, but seed storage for 18 months at room temperature proved to be a positive way to improve seed quality, especially in 'Boyiatiou'.
\end{abstract}

Keywords: Abelmoschus esculentus L., nitrogen fertilization, okra, seed hardness, seed quality

\section{Introduction}

Although several studies of the nutrient requirements of okra (Abelmoschus esculentus [L.] Moench.) have been carried out, these have been concerned primarily with the fresh crop and almost entirely refer to large-fruiting cultivars, such as 'Clemson spineless' (Asif \& Greig, 1972; Shrestha, 1983; Olasantan, 1994; Lamont, 1999). In Greece and Turkey, however, there is a market preference for small-fruiting cultivars (Duzyaman \& Vural, 2009; Koutsos, 2009) for which few data are available about their nutritional requirements and virtually none for the seed crop (Passam \& Rekoumi, 2009; Rekoumi, Karapanos, Akoumianakis, \& Passam, 2012).

Nitrogen is reported to promote the vegetative growth of plants and increase assimilation by making available more photosynthetic area (Chaurasia \& Singh, 1999; Bangar, Parmar, \& Maini, 2000). Olasantan (1999) reported that the growth and yield attributes of okra increased with increasing rates of $\mathrm{N}$. The increase, however, was proportionally greater for growth attributes than for yield characters, and when considering a seed crop, seed quality expressed in terms of 100-seed weight, viability percentage and vigour did not show significant differences due to $\mathrm{N}$ application (Singh, Gulshan, \& Jain, 1999).

Other factors affecting seed production and quality in okra include the sowing time (Yadav, Dhankar, Deshwal, \& Tomer, 2001), the prevailing environmental conditions (Anjum \& Amjad, 1999; Yadav \& Dhankhar, 1999; 
Amjad, Anjum, \& Hussain, 2001) and genetic and soil factors (Majanbu, Ogunlela, Ahmed, \& Olarewaju, 1985).

Fruit position on the plant has also been reported to influence seed quality in okra. For example, germination varied from $28-58 \%$ in upper pods, $29-87 \%$ in middle pods, and $67-83 \%$ in lower pods in different cultivars of okra (Bhatt \& Srinivasa Rao, 1998). Malik, Singh, Nehra, Khurana, Dahiya, and Singh (2000) also found that the seeds obtained from the pods of the lower and middle nodes had higher germination rates than those from the upper nodes. Seed quality may relate to the moisture content and the rate of seed drying, where low final moisture content and rapid drying promote the formation of hard seeds and reduce the total percent of seed germination (Demir, 2001; El-Balla, Saidahmed, \& Makkawi, 2011). Cabrera, Baskin, and Nsapato (1995) observed that no hard seeds were detected until seeds reached physiological maturity, while El-Balla et al. (2011) reported that okra seeds with $13 \%$ moisture content tended to show little or no seed hardness, but once the moisture content dropped to $4-6 \%$ seed hardness became prevalent. Seed hardness varies among different okra cultivars (Bennett, 1959) and is particularly high in the Greek cv. 'Boyiatiou' (Passam \& Rekoumi, 2009). It is also affected by the level of $\mathrm{N}$ application during cultivation (Rekoumi, Moustakas, Passam \& Akoumianakis, 2013).

It is possible that the rate of seed drying relates to the position of the pod on the mother plant. In view of the significance of $\mathrm{N}$ and the rate of seed drying on seed quality (especially seed hardness) two experiments were undertaken as described in this study.

\section{Material and Methods}

\subsection{Experimental Conditions}

The experiments of the present study were carried out at the Experimental Farm of the University of Thessaly, Greece during the growing season of 2011. Plants were cultivated in soil, the composition of which was $48 \%$ Sand, 29\% Silt, 23\% Clay and 1.3\% Organic matter with $\mathrm{pH} 7.9$.

\subsection{Plant Material, Treatments and Data Collection}

Seeds of four okra cultivars (e.g. 'Boyatiou', 'Veloudo', 'Pylaias' and 'Clemson') were sown in seed trays on $1^{\text {st }}$ and $20^{\text {th }}$ of May, 2011 (Experiments 1 and 2, respectively) and kept under controlled temperature $\left(20^{\circ} \mathrm{C}\right)$ until field transplantation. Plants were transplanted after 20 days in both experiments, when the plants had 3-4 true leaves, and placed in rows with $1 \mathrm{~m}$ between each row and $30 \mathrm{~cm}$ between plants within the rows. The plants were irrigated regularly on a weekly basis via a drip irrigation system. Fertilizers were applied manually at regular intervals (once a week) at a dose of $0.5 \mathrm{~L}$ per plant. $\mathrm{P}$ and $\mathrm{K}$, each were applied at a constant concentration of $150 \mathrm{mg} \mathrm{L}^{-1}$, while $\mathrm{N}$ was applied at three levels i.e. $150 \mathrm{mg} \mathrm{L}^{-1}\left(\mathrm{~F}_{1}\right.$ or control), $300 \mathrm{mg} \mathrm{L}^{-1}\left(\mathrm{~F}_{2}\right)$ and $450 \mathrm{mg} \mathrm{L}^{-1}\left(\mathrm{~F}_{3}\right)$. Side shoots were not removed and the total flower induction per plant and the number of pods set per plant (i.e. on the main stem and side shoots) were recorded.

The pods were harvested from three parts on the same plants, i.e. upper, middle and lower plant part, which were defined according to the cultivar and the total number of pods set (e.g. from 2-3 pods for each plant part for cv. 'Boyatiou' to 7-8 pods for cv. 'Clemson'). For each part, 5 pods were randomly selected from a batch sample at each harvest and the pod dimensions (length of pod - including beak, but not the peduncle- and diameter), average number of seeds per pod and 100-seed weight recorded. After harvesting the pods, seeds were either dried within the pods for 15 days (pod drying method) or after immediate extraction from the pods (seed drying method). Both methods were applied at room temperature (about $25^{\circ} \mathrm{C}$ ). Also in the pod drying method, the seed moisture content was determined prior to and after drying according to standard methods (ISTA, 2009).

Seed germination tests were conducted using 100 seeds per treatment, with four replications (400 seeds in total). The germination tests were carried out on seeds after extraction from the pods either immediately after harvest or after drying within pods for 15 days at room temperature. Just prior to the germination tests, seeds were surface sterilized firstly with Chlorine Dioxide, 1 to 20 parts deionized water for $10 \mathrm{sec}$. and then with Mancozeb fungicide $(80 \% \mathrm{w} / \mathrm{w}$ diluted in distilled water at a concentration of $2 \mathrm{~g}$ per liter) and placed in sterilized Petri dishes on a double layer of Whatman No. 1 filter paper moistened with distilled water. The Petri dishes were placed in the dark in an incubator at $25^{\circ} \mathrm{C}$ for 21 days and every 2 days germinated seeds were counted and removed and, in the case of moisture deficiency, distilled water was added. Seeds were considered germinated when the tip of the radical had grown free of the seed coat (Wiese \& Binning, 1987; Auld, Bettis, Crock, \& Kephart, 1988). Those seeds which did not germinate (not hydrated) and remained rigid after the time period of 21 days in the incubator were considered to be hard seeds, but without testing by scarification (Passam \& Polyzou, 1997) or another means, e.g. tetrazolium (Moore, 1985). Germination percentage was calculated by the following formula (ISTA, 2009). 


$$
\text { Germination }(\%)=\frac{\text { Number of seeds germinated }}{\text { Number of seeds tested }} \times 100
$$

Seeds were also stored at room temperature for a period of 18 months and tested for their germination potential at the end of storage.

\subsection{Statistical Analysis}

The experiment was laid out in a Randomized Complete Block design with factorial arrangements using three replications $(3 \times 12=36$ plots and 10 plants in each plot). Statistical analysis was carried out by using SAS statistical package software (SAS Institute Inc., USA) and the means were compared by employing least significant difference (LSD) test at $p<0.05$. Graphs were generated by using Microsoft Office Excel software (Microsoft Corporation, USA).

\section{Results}

\subsection{Harvesting and Climatic Conditions}

The climatic conditions of the experimental site are given in Figure 1. The temperature gradually increased from May to July and then decreased till October. There was a difference of about $12-16{ }^{\circ} \mathrm{C}$ between minimum and maximum temperatures (in mean overall differences) throughout the experimental period. There was no rainfall during July, but during the other months varied from 23 to $43 \mathrm{~mm}$; relative humidity thus decreased during July, but afterwards increased progressively. This means that as the number of days from anthesis increased (from 30-50 DAA) pods were increasingly exposed to lower temperatures and higher humidity.

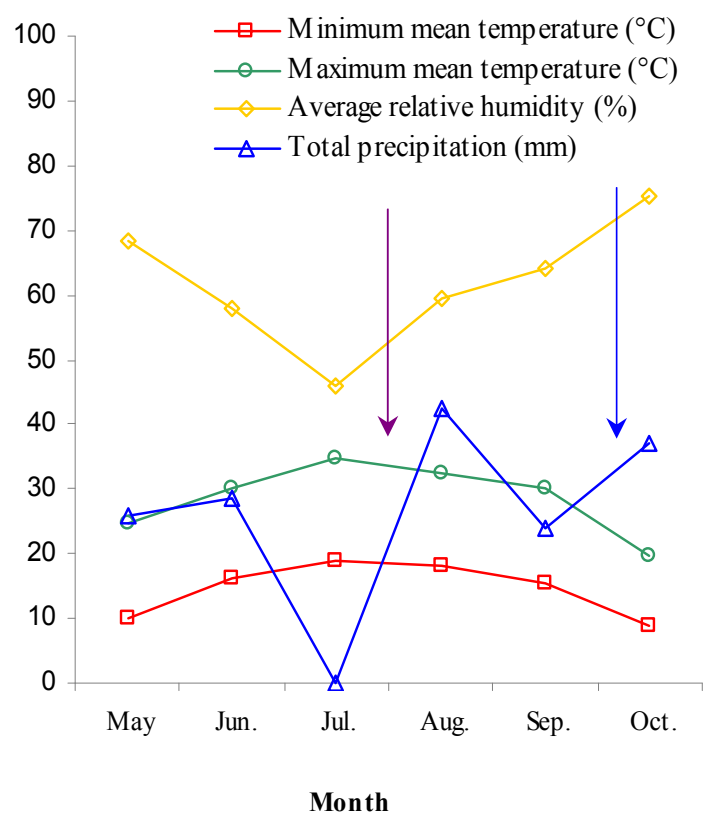

Figure 1. Climate conditions of the experimental site during the cultivation period 2011 (vertical arrows indicate the start and finish point of pod harvest)

\subsection{Total Flower Induction}

In both experiments, flower induction was significantly affected by the $\mathrm{N}$ level in all the cultivars studied, except cv. 'Pylaias' (Table 1). At all the N levels, 'Clemson' produced a higher number of flowers per plant than the other cultivars. Except in 'Boyiatiou', the flower induction of which was very low at 300 ppm N, in both experiments the best $\mathrm{N}$ rates were 150 and $300 \mathrm{ppm} \mathrm{N}$. Between cultivars, 'Boyiatiou' produced the lowest number of flowers at all $\mathrm{N}$ levels, whereas 'Clemson' produced the highest number of flowers (Figure 2). 
Table 1. Effect of $\mathrm{N}$ levels on flower induction and pod set of four okra cultivars

\begin{tabular}{|c|c|c|c|c|c|}
\hline \multirow[b]{2}{*}{ Cultivar (C) } & \multirow{2}{*}{$\begin{array}{l}\text { N level } \\
\left(\mathrm{mg} \mathrm{l}^{-1}\right)(\mathrm{F})\end{array}$} & \multicolumn{2}{|c|}{ Total flower induction (flowers/plant) } & \multicolumn{2}{|c|}{ Pod set (pods/plant) } \\
\hline & & $\begin{array}{l}\text { Experiment } 1 \\
\text { ( } 1^{\text {st }} \text { of May) }\end{array}$ & $\begin{array}{l}\text { Experiment } 2 \\
\left(20^{\text {th }} \text { of May) }\right.\end{array}$ & $\begin{array}{l}\text { Experiment } 1 \\
\left(1^{\text {st }} \text { of May }\right)\end{array}$ & $\begin{array}{l}\text { Experiment } 2 \\
\left(20^{\text {th }} \text { of May) }\right.\end{array}$ \\
\hline \multirow{3}{*}{ 'Boyiatiou' } & $150\left(\mathrm{~F}_{1}\right)$ & $14.8^{\mathrm{a}}$ & $13.0^{\mathrm{a}}$ & $5.5^{\mathrm{b}}$ & $4.8^{\mathrm{b}}$ \\
\hline & $300\left(\mathrm{~F}_{2}\right)$ & $8.6^{\mathrm{b}}$ & $7.0^{\mathrm{b}}$ & $5.0^{\mathrm{b}}$ & $4.2^{\mathrm{b}}$ \\
\hline & $450\left(\mathrm{~F}_{3}\right)$ & $13.4^{\mathrm{a}}$ & $11.8^{\mathrm{a}}$ & $11.5^{\mathrm{a}}$ & $10.0^{\mathrm{a}}$ \\
\hline Mean & & 12.2 & 10.6 & 7.3 & 6.3 \\
\hline$L S D$ & & 2.6 & 2.7 & 2.5 & 2.4 \\
\hline \multirow{3}{*}{ 'Veloudo' } & $150\left(\mathrm{~F}_{1}\right)$ & $20.5^{\mathrm{ab}}$ & $18.9^{\mathrm{ab}}$ & $18.4^{\mathrm{ab}}$ & $15.0^{\mathrm{a}}$ \\
\hline & $300\left(\mathrm{~F}_{2}\right)$ & $22.9^{\mathrm{a}}$ & $21.0^{\mathrm{a}}$ & $20.9^{\mathrm{a}}$ & $16.9^{\mathrm{a}}$ \\
\hline & $450\left(\mathrm{~F}_{3}\right)$ & $17.8^{\mathrm{b}}$ & $16.0^{\mathrm{b}}$ & $16.8^{\mathrm{b}}$ & $13.8^{\mathrm{a}}$ \\
\hline Mean & & 20.4 & 18.6 & 18.7 & 15.2 \\
\hline$L S D$ & & 4.3 & 4.4 & 2.7 & 3.4 \\
\hline \multirow{3}{*}{ 'Pylaias' } & $150\left(\mathrm{~F}_{1}\right)$ & $23.9^{\mathrm{a}}$ & $21.0^{\mathrm{a}}$ & $22.6^{\mathrm{a}}$ & $19.9^{\mathrm{a}}$ \\
\hline & $300\left(\mathrm{~F}_{2}\right)$ & $23.3^{\mathrm{a}}$ & $21.0^{\mathrm{a}}$ & $21.9^{\mathrm{a}}$ & $17.9^{\mathrm{a}}$ \\
\hline & $450\left(\mathrm{~F}_{3}\right)$ & $18.9^{\mathrm{a}}$ & $16.4^{\mathrm{a}}$ & $18.0^{\mathrm{a}}$ & $14.9^{\mathrm{a}}$ \\
\hline Mean & & 22.0 & 19.4 & 20.8 & 17.5 \\
\hline$L S D$ & & 7.5 & 7.6 & 4.7 & 6.2 \\
\hline \multirow{3}{*}{ 'Clemson' } & $150\left(\mathrm{~F}_{1}\right)$ & $26.8^{\mathrm{a}}$ & $24.8^{\mathrm{a}}$ & $24.8^{\mathrm{ab}}$ & $21.6^{\mathrm{b}}$ \\
\hline & $300\left(\mathrm{~F}_{2}\right)$ & $29.6^{\mathrm{a}}$ & $26.9^{\mathrm{a}}$ & $28.0^{\mathrm{a}}$ & $23.7^{\mathrm{a}}$ \\
\hline & $450\left(\mathrm{~F}_{3}\right)$ & $20.5^{\mathrm{b}}$ & $18.7^{\mathrm{b}}$ & $20.3^{b}$ & $18.5^{\mathrm{b}}$ \\
\hline Mean & & 25.6 & 23.4 & 24.3 & 21.2 \\
\hline$L S D$ & & 6.0 & 5.9 & 3.9 & 5.1 \\
\hline \multicolumn{6}{|l|}{$\mathrm{C} \times \mathrm{F}$} \\
\hline$\left(\mathrm{C} \times \mathrm{F}_{1}\right)$ & & $*$ & $*$ & $*$ & $*$ \\
\hline$\left(\mathrm{C} \times \mathrm{F}_{2}\right)$ & & $*$ & $*$ & * & $*$ \\
\hline$\left(\mathrm{C} \times \mathrm{F}_{3}\right)$ & & $*$ & $*$ & $*$ & $*$ \\
\hline
\end{tabular}

Mean separation by LSD test. Mean values in the same column for each cultivar separately followed by different letters differ significantly at $p=0.05 . *=$ statistically significant $(p<0.05)$. 


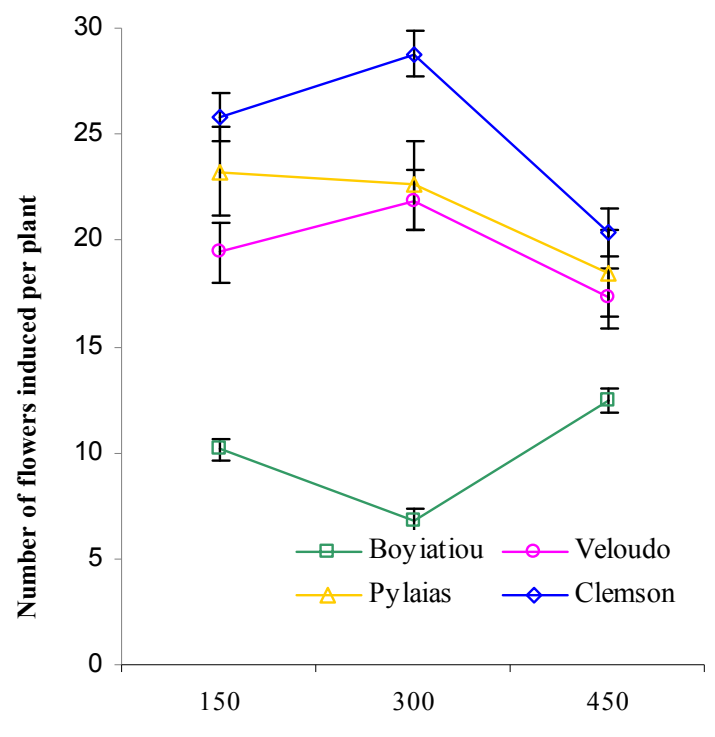

N level $\left(\mathrm{mg} \mathrm{L}^{-1}\right)$

Figure 2. Effect of $\mathrm{N}$ levels on the total flower induction of four okra cultivars

\subsection{Pod Set}

In both experiments, the $\mathrm{N}$ level had a significant effect on pod set in all the cultivars except 'Pylaias' (both experiments) and 'Veloudo' (Experiment 2, only). In 'Boyiatiou' pod set was highest at $450 \mathrm{ppm} \mathrm{N}$, whereas in the other cultivars pod set was reduced at this $\mathrm{N}$ concentration, although not always to a statistically significant extent (Table 1). No differences in pod set were detected between $\mathrm{N}$ levels of 150 and $300 \mathrm{ppm}$ in any of the cultivars, except 'Clemson' where, in Experiment 2, pod set was higher at $300 \mathrm{ppm} \mathrm{N}$ than at $150 \mathrm{ppm} \mathrm{N}$ (Table 1, Figure 3).

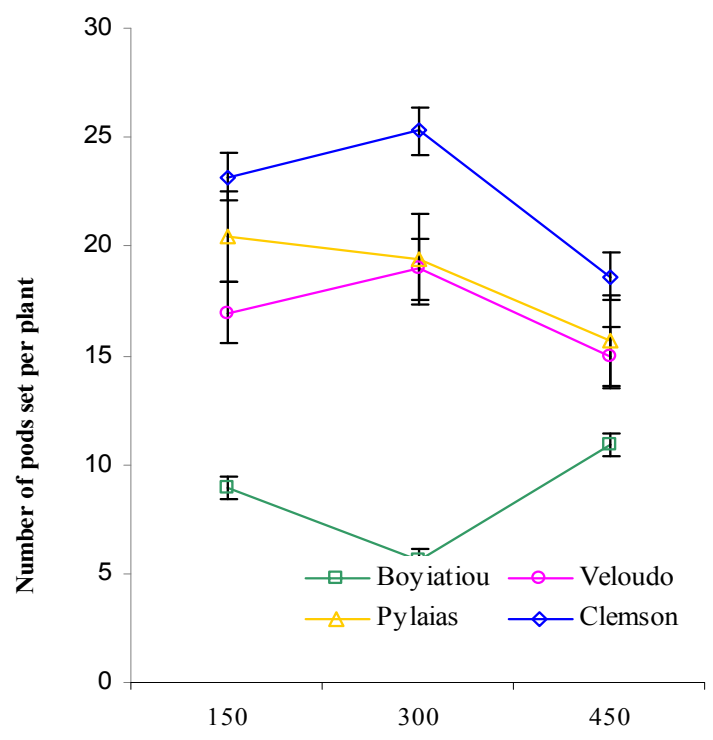

$\mathrm{N}$ level ( $\left.\mathrm{mg} \mathrm{L}^{-1}\right)$

Figure 3. Effect of $\mathrm{N}$ levels on the number of pods of four okra cultivars 


\subsection{Pod Length}

Overall, in Experiment 1 pod length was significantly higher for pods harvested from the middle region of the plant than those at the lower or higher regions (Table 2), whereas in Experiment 2 the same trend was observed (except in 'Boyiatiou'), but to a non-significant degree (Table 3). In Experiment 1, with few exceptions (Pylaias in the middle region and Clemson in both the middle and lower regions) there was virtually no effect of $\mathrm{N}$ on pod length, irrespective of the position of the pods on the plant. Similarly in Experiment 2, N level did not affect pod length in the majority of cases, irrespective of cultivar and pod position on the plant.

Table 2. Effect of $\mathrm{N}$ levels on the length and diameter of pods harvested from different plant parts of four okra cultivars (Experiment 1)

\begin{tabular}{|c|c|c|c|c|c|c|c|c|c|}
\hline \multirow{2}{*}{ Cultivar (C) } & \multirow{2}{*}{$\begin{array}{l}\text { N level } \\
\left(\mathrm{mg} \mathrm{l}^{-1}\right)(\mathrm{F})\end{array}$} & \multicolumn{3}{|c|}{ Pod length $(\mathrm{cm})$} & \multirow{2}{*}{ LSD } & \multicolumn{3}{|c|}{ Pod diameter $(\mathrm{cm})$} & \multirow{2}{*}{ LSD } \\
\hline & & Upper & Middle & Lower & & Upper & Middle & Lower & \\
\hline \multirow{3}{*}{ 'Boyiatiou' } & $150\left(\mathrm{~F}_{1}\right)$ & $17.8^{\mathrm{a}(\mathrm{b})}$ & $18.9^{\mathrm{a}(\mathrm{a})}$ & $15.2^{\mathrm{a}(\mathrm{c})}$ & 1.4 & $2.2^{\mathrm{a}(\mathrm{a})}$ & $2.3^{\mathrm{a}(\mathrm{a})}$ & $2.0^{\mathrm{a}(\mathrm{a})}$ & 0.4 \\
\hline & $300\left(\mathrm{~F}_{2}\right)$ & $17.2^{\mathrm{ab}(\mathrm{ab})}$ & $18.8^{\mathrm{a}(\mathrm{a})}$ & $16.3^{\mathrm{a}(\mathrm{b})}$ & 1.9 & $2.1^{\mathrm{ab}(\mathrm{a})}$ & $2.3^{\mathrm{a}(\mathrm{a})}$ & $2.2^{\mathrm{a}(\mathrm{a})}$ & 0.2 \\
\hline & $450\left(\mathrm{~F}_{3}\right)$ & $16.2^{\mathrm{b}(\mathrm{b})}$ & $19.3^{\mathrm{a}(\mathrm{a})}$ & $16.6^{\mathrm{a}(\mathrm{b})}$ & 1.9 & $1.9^{\mathrm{b}(\mathrm{b})}$ & $2.3^{\mathrm{a}(\mathrm{a})}$ & $2.2^{\mathrm{a}(\mathrm{a})}$ & 0.1 \\
\hline Mean & & 17.0 & 19.0 & 16.0 & & 2.0 & 2.3 & 2.1 & \\
\hline$L S D$ & & 1.4 & 0.8 & 2.6 & & 0.2 & 0.2 & 0.3 & \\
\hline \multirow{3}{*}{ 'Veloudo' } & $150\left(\mathrm{~F}_{1}\right)$ & $20.1^{\mathrm{a}(\mathrm{b})}$ & $24.1^{\mathrm{a}(\mathrm{a})}$ & $20.8^{\mathrm{a}(\mathrm{b})}$ & 1.4 & $2.0^{\mathrm{a}(\mathrm{b})}$ & $2.2^{\mathrm{a}(\mathrm{ab})}$ & $2.3^{\mathrm{a}(\mathrm{a})}$ & 0.2 \\
\hline & $300\left(\mathrm{~F}_{2}\right)$ & $19.9^{\mathrm{a}(\mathrm{c})}$ & $24.5^{\mathrm{a}(\mathrm{a})}$ & $21.5^{\mathrm{a}(\mathrm{b})}$ & 0.6 & $2.0^{\mathrm{a}(\mathrm{a})}$ & $2.4^{\mathrm{a}(\mathrm{a})}$ & $2.3^{\mathrm{a}(\mathrm{a})}$ & 3.5 \\
\hline & $450\left(\mathrm{~F}_{3}\right)$ & $21.0^{\mathrm{a}(\mathrm{b})}$ & $23.9^{\mathrm{a}(\mathrm{a})}$ & $20.5^{\mathrm{a}(\mathrm{b})}$ & 1.4 & $2.0^{\mathrm{a}(\mathrm{b})}$ & $2.3^{\mathrm{a}(\mathrm{a})}$ & $2.2^{\mathrm{a}(\mathrm{ab})}$ & 0.2 \\
\hline Mean & & 20.3 & 24.1 & 20.9 & & 2.0 & 2.3 & 2.2 & \\
\hline$L S D$ & & 1.2 & 0.9 & 1.4 & & 0.2 & 0.2 & 0.3 & \\
\hline \multirow{3}{*}{ 'Pylaias' } & $150\left(\mathrm{~F}_{1}\right)$ & $17.9^{\mathrm{a}(\mathrm{b})}$ & $20.7^{\mathrm{a}(\mathrm{a})}$ & $18.1^{\mathrm{a}(\mathrm{b})}$ & 1.6 & $2.0^{\mathrm{a}(\mathrm{a})}$ & $1.8^{\mathrm{a}(\mathrm{b})}$ & $1.9^{\mathrm{ab}(\mathrm{ab})}$ & 0.1 \\
\hline & $300\left(\mathrm{~F}_{2}\right)$ & $19.9^{\mathrm{a}(\mathrm{a})}$ & $19.2^{\mathrm{b}(\mathrm{a})}$ & $18.2^{\mathrm{a}(\mathrm{a})}$ & 2.6 & $1.9^{\mathrm{b}(\mathrm{a})}$ & $1.6^{\mathrm{a}(\mathrm{b})}$ & $1.8^{\mathrm{b}(\mathrm{ab})}$ & 0.2 \\
\hline & $450\left(\mathrm{~F}_{3}\right)$ & $18.2^{\mathrm{a}(\mathrm{ab})}$ & $19.2^{\mathrm{b}(\mathrm{a})}$ & $17.5^{\mathrm{a}(\mathrm{b})}$ & 1.4 & $2.1^{\mathrm{a}(\mathrm{a})}$ & $1.7^{\mathrm{a}(\mathrm{b})}$ & $2.0^{\mathrm{a}(\mathrm{a})}$ & 0.2 \\
\hline Mean & & 18.6 & 19.7 & 17.9 & & 2.0 & 1.7 & 1.9 & \\
\hline$L S D$ & & 2.5 & 0.6 & 2.2 & & 0.2 & 0.2 & 0.1 & \\
\hline \multirow{3}{*}{ 'Clemson' } & $150\left(\mathrm{~F}_{1}\right)$ & $15.6^{\mathrm{a}(\mathrm{ab})}$ & $15.3^{\mathrm{b}(\mathrm{b})}$ & $15.9^{\mathrm{ab}(\mathrm{a})}$ & 0.4 & $1.6^{\mathrm{a}(\mathrm{b})}$ & $1.6^{\mathrm{a}(\mathrm{b})}$ & $1.8^{\mathrm{a}(\mathrm{a})}$ & 0.1 \\
\hline & $300\left(F_{2}\right)$ & $14.9^{\mathrm{a}(\mathrm{b})}$ & $17.0^{\mathrm{a}(\mathrm{a})}$ & $14.5^{\mathrm{b}(\mathrm{b})}$ & 1.3 & $1.6^{\mathrm{a}(\mathrm{a})}$ & $1.6^{\mathrm{a}(\mathrm{a})}$ & $1.8^{\mathrm{a}(\mathrm{a})}$ & 0.3 \\
\hline & $450\left(\mathrm{~F}_{3}\right)$ & $15.6^{\mathrm{a}(\mathrm{a})}$ & $16.9^{\mathrm{a}(\mathrm{a})}$ & $16.4^{\mathrm{a}(\mathrm{a})}$ & 1.7 & $1.7^{\mathrm{a}(\mathrm{a})}$ & $1.7^{\mathrm{a}(\mathrm{a})}$ & $1.9^{\mathrm{a}(\mathrm{a})}$ & 0.3 \\
\hline Mean & & 15.3 & 16.4 & 15.6 & & 1.6 & 1.6 & 1.8 & \\
\hline$L S D$ & & 0.9 & 1.0 & 1.7 & & 0.2 & 0.2 & 0.1 & \\
\hline \multicolumn{10}{|l|}{$\mathrm{C} \times \mathrm{F}$} \\
\hline$\left(\mathrm{C} \times \mathrm{F}_{1}\right)$ & & $*$ & * & * & & $*$ & $*$ & $*$ & \\
\hline$\left(\mathrm{C} \times \mathrm{F}_{2}\right)$ & & $*$ & * & * & & $*$ & * & $*$ & \\
\hline$\left(\mathrm{C} \times \mathrm{F}_{3}\right)$ & & $*$ & $*$ & $*$ & & $*$ & $*$ & $*$ & \\
\hline
\end{tabular}

Mean separation by LSD test. Mean values in the same column for each cultivar separately followed by different letters differ significantly at $p=0.05$. Means in each row for each pod characteristic separately followed by the same letter in parenthesis do not differ significantly at $p=0.05$.

- = statistically significant $(p<0.05)$. 
Table 3. Effect of $\mathrm{N}$ levels on the length and diameter of pods harvested from different plant parts of four okra cultivars (Experiment 2)

\begin{tabular}{|c|c|c|c|c|c|c|c|c|c|}
\hline \multirow{2}{*}{ Cultivar (C) } & \multirow{2}{*}{$\begin{array}{l}\text { N level } \\
\left(\mathrm{mg} \mathrm{l}^{-1}\right)(\mathrm{F})\end{array}$} & \multicolumn{3}{|c|}{ Pod length $(\mathrm{cm})$} & \multirow{2}{*}{ LSD } & \multicolumn{3}{|c|}{ Pod diameter $(\mathrm{cm})$} & \multirow{2}{*}{ LSD } \\
\hline & & Upper & Middle & Lower & & Upper & Middle & Lower & \\
\hline \multirow{3}{*}{ 'Boyiatiou' } & $150\left(\mathrm{~F}_{1}\right)$ & $17.4^{\mathrm{b}(\mathrm{b})}$ & $17.8^{\mathrm{a}(\mathrm{ab})}$ & $18.6^{\mathrm{a}(\mathrm{a})}$ & 0.9 & $2.1^{\mathrm{a}(\mathrm{a})}$ & $2.2^{\mathrm{a}(\mathrm{a})}$ & $2.1^{\mathrm{a}(\mathrm{a})}$ & 0.2 \\
\hline & $300\left(\mathrm{~F}_{2}\right)$ & $19.9^{\mathrm{a}(\mathrm{a})}$ & $17.5^{\mathrm{a}(\mathrm{b})}$ & $16.6^{\mathrm{b}(\mathrm{b})}$ & 1.6 & $2.3^{\mathrm{a}(\mathrm{a})}$ & $2.1^{\mathrm{a}(\mathrm{a})}$ & $2.1^{\mathrm{a}(\mathrm{a})}$ & 0.2 \\
\hline & $450\left(\mathrm{~F}_{3}\right)$ & $19.2^{\mathrm{ab}(\mathrm{a})}$ & $18.4^{\mathrm{a}(\mathrm{a})}$ & $18.2^{\mathrm{a}(\mathrm{a})}$ & 1.4 & $2.2^{\mathrm{a}(\mathrm{ab})}$ & $2.3^{\mathrm{a}(\mathrm{a})}$ & $2.0^{\mathrm{a}(\mathrm{b})}$ & 0.2 \\
\hline Mean & & 18.8 & 17.9 & 17.8 & & 2.2 & 2.2 & 2.0 & \\
\hline$L S D$ & & 2.0 & 0.9 & 0.8 & & 0.2 & 0.2 & 0.2 & \\
\hline \multirow{3}{*}{ 'Veloudo' } & $150\left(\mathrm{~F}_{1}\right)$ & $18.7^{\mathrm{a}(\mathrm{c})}$ & $24.0^{\mathrm{a}(\mathrm{a})}$ & $21.9^{\mathrm{ab}(\mathrm{b})}$ & 2.0 & $1.7^{\mathrm{a}(\mathrm{b})}$ & $2.4^{\mathrm{a}(\mathrm{a})}$ & $2.2^{\mathrm{a}(\mathrm{a})}$ & 0.3 \\
\hline & $300\left(\mathrm{~F}_{2}\right)$ & $20.8^{\mathrm{a}(\mathrm{b})}$ & $21.7^{\mathrm{b}(\mathrm{ab})}$ & $23.4^{\mathrm{a}(\mathrm{a})}$ & 2.5 & $2.2^{\mathrm{a}(\mathrm{a})}$ & $2.2^{\mathrm{b}(\mathrm{a})}$ & $2.3^{\mathrm{a}(\mathrm{a})}$ & 0.3 \\
\hline & $450\left(\mathrm{~F}_{3}\right)$ & $19.8^{\mathrm{a}(\mathrm{b})}$ & $22.9^{\mathrm{ab}(\mathrm{a})}$ & $20.9^{b(a b)}$ & 2.1 & $2.0^{\mathrm{a}(\mathrm{b})}$ & $2.4^{\mathrm{a}(\mathrm{a})}$ & $2.3^{\mathrm{a}(\mathrm{ab})}$ & 0.3 \\
\hline Mean & & 19.7 & 22.8 & 22.0 & & 1.9 & 2.3 & 2.2 & \\
\hline LSD & & 2.1 & 2.2 & 2.4 & & 0.3 & 0.1 & 0.4 & \\
\hline \multirow{3}{*}{ 'Pylaias' } & $150\left(\mathrm{~F}_{1}\right)$ & $18.9^{\mathrm{a}(\mathrm{ab})}$ & $20.4^{\mathrm{ab}(\mathrm{a})}$ & $17.1^{\mathrm{b}(\mathrm{b})}$ & 2.2 & $1.8^{\mathrm{a}(\mathrm{ab})}$ & $2.0^{\mathrm{ab}(\mathrm{a})}$ & $1.7^{\mathrm{a}(\mathrm{b})}$ & 0.2 \\
\hline & $300\left(\mathrm{~F}_{2}\right)$ & $19.5^{\mathrm{a}(\mathrm{b})}$ & $21.4^{\mathrm{a}(\mathrm{a})}$ & $19.7^{\mathrm{a}(\mathrm{b})}$ & 1.6 & $2.1^{\mathrm{a}(\mathrm{a})}$ & $2.1^{\mathrm{a}(\mathrm{a})}$ & $1.7^{\mathrm{a}(\mathrm{b})}$ & 0.3 \\
\hline & $450\left(\mathrm{~F}_{3}\right)$ & $19.7^{\mathrm{a}(\mathrm{a})}$ & $18.5^{\mathrm{b}(\mathrm{ab})}$ & $17.2^{\mathrm{b}(\mathrm{b})}$ & 1.3 & $1.9^{\mathrm{a}(\mathrm{a})}$ & $1.8^{\mathrm{b}(\mathrm{ab})}$ & $1.6^{\mathrm{a}(\mathrm{b})}$ & 0.2 \\
\hline Mean & & 19.3 & 20.1 & 18.0 & & 1.9 & 1.9 & 1.6 & \\
\hline$L S D$ & & 2.0 & 1.3 & 1.9 & & 0.3 & 0.2 & 0.2 & \\
\hline \multirow{3}{*}{ 'Clemson' } & $150\left(\mathrm{~F}_{1}\right)$ & $16.0^{\mathrm{b}(\mathrm{a})}$ & $16.9^{a(a)}$ & $16.2^{\mathrm{b}(\mathrm{a})}$ & 0.8 & $1.6^{\mathrm{b}(\mathrm{a})}$ & $1.5^{\mathrm{a}(\mathrm{ab})}$ & $1.4^{\mathrm{a}(\mathrm{b})}$ & 0.1 \\
\hline & $300\left(\mathrm{~F}_{2}\right)$ & $16.5^{\mathrm{b}(\mathrm{a})}$ & $17.0^{\mathrm{a}(\mathrm{a})}$ & $17.3^{\mathrm{a}(\mathrm{a})}$ & 0.8 & $1.5^{\mathrm{b}(\mathrm{a})}$ & $1.5^{\mathrm{a}(\mathrm{a})}$ & $1.5^{\mathrm{a}(\mathrm{a})}$ & 0.1 \\
\hline & $450\left(\mathrm{~F}_{3}\right)$ & $19.2^{\mathrm{a}(\mathrm{a})}$ & $16.9^{\mathrm{a}(\mathrm{b})}$ & $17.5^{\mathrm{a}(\mathrm{ab})}$ & 2.1 & $1.9^{\mathrm{a}(\mathrm{a})}$ & $1.4^{\mathrm{a}(\mathrm{b})}$ & $1.6^{\mathrm{a}(\mathrm{ab})}$ & 0.3 \\
\hline Mean & & 17.2 & 16.9 & 17.0 & & 1.6 & 1.4 & 1.5 & \\
\hline$L S D$ & & 2.1 & 0.8 & 0.9 & & 0.2 & 0.1 & 0.2 & \\
\hline \multicolumn{10}{|l|}{$\mathrm{C} \times \mathrm{F}$} \\
\hline$\left(\mathrm{C} \times \mathrm{F}_{1}\right)$ & & $*$ & $*$ & $*$ & & $*$ & $*$ & $*$ & \\
\hline$\left(\mathrm{C} \times \mathrm{F}_{2}\right)$ & & $*$ & $*$ & $*$ & & $*$ & * & $*$ & \\
\hline$\left(\mathrm{C} \times \mathrm{F}_{3}\right)$ & & ns & $*$ & $*$ & & ns & $*$ & $*$ & \\
\hline
\end{tabular}

Mean separation by LSD test. Mean values in the same column for each cultivar separately followed by different letters differ significantly at $p=0.05$. Means in each row for each pod characteristic separately followed by the same letter in parenthesis do not differ significantly at $p=0.05$.

$*=$ statistically significant $(p<0.05)$.

\subsection{Pod Diameter}

Pod diameter was not significantly affected by the pod position on plant in either experiment and overall ranged from 1.6-2.3 cm (Experiment 1) and 1.4-2.3 cm (Experiment 2) (Tables 2-3). In addition, with few exceptions (e.g. middle pods of Veloudo and upper pods of Clemson in Experiment 2) pod diameter was not significantly affected by the $\mathrm{N}$ levels (Tables 2-3).

Overall, in both experiments the pod diameter of cv. 'Clemson, was lower than that of the other cultivars, irrespective of $\mathrm{N}$ level and the position of the pods on the plant.

\subsection{Number of Seeds per Pod}

The number of seeds per pod was higher in the pods from the middle and upper part of the plant in 'Clemson' and 'Pylaias', as well as in 'Boyiatiou' (at $450 \mathrm{ppm} \mathrm{N}$ ) and 'Veloudo' (at $150 \mathrm{ppm} \mathrm{N}$ ) in Experiment 1 (Table 4), but only in 'Veloudo' (350 and $450 \mathrm{ppm} \mathrm{N}$ ) in Experiment 2 (Table 5). 
The $\mathrm{N}$ level had no significant effect on the number of seeds per pod in any cultivar in either experiment, except 'Veloudo' where seed number was lower at $150 \mathrm{ppm} \mathrm{N}$ than $450 \mathrm{ppm} \mathrm{N}$ in the lower pods (Experiment 1) and upper pods (Experiment 2), but higher in 'Pylaias' (middle pods) and 'Clemson' (lower pods) in Experiment 1 and in 'Veloudo' (middle pods) in Experiment 2.

Table 4. Effect of $\mathrm{N}$ levels on seed characteristics from pods harvested from different plant parts of four okra cultivars (Experiment 1)

\begin{tabular}{|c|c|c|c|c|c|c|c|c|c|}
\hline \multirow{2}{*}{ Cultivar (C) } & \multirow{2}{*}{$\begin{array}{l}\text { N level } \\
\left(\mathrm{mg} \mathrm{l}^{-1}\right)(\mathrm{F})\end{array}$} & \multicolumn{3}{|c|}{ Number of seeds/pod } & \multirow{2}{*}{ LSD } & \multicolumn{3}{|c|}{ 100-seed weight (g) } & \multirow{2}{*}{ LSD } \\
\hline & & Upper & Middle & Lower & & Upper & Middle & Lower & \\
\hline \multirow{3}{*}{ 'Boyiatiou' } & $150\left(\mathrm{~F}_{1}\right)$ & $72.7^{\mathrm{a}(\mathrm{a})}$ & $64.4^{\mathrm{a}(\mathrm{b})}$ & $67.4^{\mathrm{a}(\mathrm{ab})}$ & 6.9 & $28.9^{\mathrm{a}(\mathrm{a})}$ & $26.2^{\mathrm{a}(\mathrm{a})}$ & $28.2^{\mathrm{a}(\mathrm{a})}$ & 7.3 \\
\hline & $300\left(F_{2}\right)$ & $68.2^{\mathrm{a}(\mathrm{a})}$ & $63.2^{\mathrm{a}(\mathrm{a})}$ & $68.4^{\mathrm{a}(\mathrm{a})}$ & 8.8 & $25.1^{\mathrm{a}(\mathrm{a})}$ & $28.1^{\mathrm{a}(\mathrm{a})}$ & $24.7^{\mathrm{a}(\mathrm{a})}$ & 6.4 \\
\hline & $450\left(\mathrm{~F}_{3}\right)$ & $73.2^{\mathrm{a}(\mathrm{a})}$ & $68.3^{\mathrm{a}(\mathrm{ab})}$ & $62.7^{\mathrm{a}(\mathrm{b})}$ & 5.7 & $26.5^{\mathrm{a}(\mathrm{a})}$ & $26.8^{\mathrm{a}(\mathrm{a})}$ & $29.4^{\mathrm{a}(\mathrm{a})}$ & 7.9 \\
\hline Mean & & 71.3 & 65.3 & 66.1 & & 26.8 & 27.0 & 27.4 & \\
\hline$L S D$ & & 19.4 & 8.1 & 6.6 & & 10.4 & 10.7 & 11.7 & \\
\hline \multirow{3}{*}{ 'Veloudo' } & $150\left(\mathrm{~F}_{1}\right)$ & $71.5^{\mathrm{a}(\mathrm{a})}$ & $57.9^{\mathrm{a}(\mathrm{b})}$ & $48.0^{\mathrm{b}(\mathrm{b})}$ & 10.1 & $34.5^{\mathrm{a}(\mathrm{a})}$ & $35.9^{\mathrm{a}(\mathrm{a})}$ & $34.4^{\mathrm{a}(\mathrm{a})}$ & 8.2 \\
\hline & $300\left(\mathrm{~F}_{2}\right)$ & $62.8^{\mathrm{a}(\mathrm{a})}$ & $56.3^{\mathrm{a}(\mathrm{a})}$ & $59.8^{\mathrm{a}(\mathrm{a})}$ & 14.6 & $35.3^{\mathrm{a}(\mathrm{a})}$ & $35.7^{\mathrm{a}(\mathrm{a})}$ & $31.2^{\mathrm{a}(\mathrm{a})}$ & 8.1 \\
\hline & $450\left(\mathrm{~F}_{3}\right)$ & $70.7^{\mathrm{a}(\mathrm{a})}$ & $60.8^{\mathrm{a}(\mathrm{a})}$ & $61.4^{\mathrm{a}(\mathrm{a})}$ & 11.2 & $34.0^{\mathrm{a}(\mathrm{a})}$ & $34.6^{\mathrm{a}(\mathrm{a})}$ & $32.0^{\mathrm{a}(\mathrm{a})}$ & 8.6 \\
\hline Mean & & 68.3 & 58.3 & 56.4 & & 34.6 & 35.4 & 32.5 & \\
\hline$L S D$ & & 16.4 & 8.4 & 6.1 & & 10.6 & 10.3 & 13.0 & \\
\hline \multirow{3}{*}{ 'Pylaias' } & $150\left(\mathrm{~F}_{1}\right)$ & $76.7^{\mathrm{a}(\mathrm{ab})}$ & $88.3^{\mathrm{a}(\mathrm{a})}$ & $60.8^{\mathrm{a}(\mathrm{b})}$ & 15.8 & $31.8^{\mathrm{a}(\mathrm{a})}$ & $28.9^{\mathrm{a}(\mathrm{a})}$ & $28.3^{\mathrm{a}(\mathrm{a})}$ & 5.4 \\
\hline & $300\left(\mathrm{~F}_{2}\right)$ & $74.7^{\mathrm{a}(\mathrm{ab})}$ & $83.4^{\mathrm{ab}(\mathrm{a})}$ & $63.7^{\mathrm{a}(\mathrm{b})}$ & 16.4 & $35.0^{\mathrm{a}(\mathrm{a})}$ & $29.8^{\mathrm{a}(\mathrm{a})}$ & $33.5^{\mathrm{a}(\mathrm{a})}$ & 9.5 \\
\hline & $450\left(\mathrm{~F}_{3}\right)$ & $77.5^{\mathrm{a}(\mathrm{a})}$ & $71.4^{\mathrm{b}(\mathrm{a})}$ & $64.8^{\mathrm{a}(\mathrm{a})}$ & 14.0 & $36.4^{\mathrm{a}(\mathrm{a})}$ & $31.1^{\mathrm{a}(\mathrm{a})}$ & $30.6^{\mathrm{a}(\mathrm{a})}$ & 8.2 \\
\hline Mean & & 76.3 & 81.0 & 63.1 & & 34.4 & 29.9 & 30.8 & \\
\hline$L S D$ & & 9.3 & 10.5 & 15.4 & & 12.3 & 10.8 & 11.5 & \\
\hline \multirow{3}{*}{ 'Clemson' } & $150\left(\mathrm{~F}_{1}\right)$ & $92.2^{\mathrm{a}(\mathrm{a})}$ & $92.4^{\mathrm{a}(\mathrm{a})}$ & $78.7^{\mathrm{a}(\mathrm{a})}$ & 16.9 & $30.0^{\mathrm{a}(\mathrm{a})}$ & $30.5^{\mathrm{a}(\mathrm{a})}$ & $30.6^{\mathrm{a}(\mathrm{a})}$ & 8.4 \\
\hline & $300\left(F_{2}\right)$ & $99.2^{\mathrm{a}(\mathrm{a})}$ & $92.7^{\text {a(a) }}$ & $71.4^{\mathrm{ab}(\mathrm{b})}$ & 14.6 & $30.0^{\mathrm{a}(\mathrm{a})}$ & $29.3^{\mathrm{a}(\mathrm{a})}$ & $27.4^{a(a)}$ & 5.6 \\
\hline & $450\left(\mathrm{~F}_{3}\right)$ & $92.2^{\mathrm{a}(\mathrm{a})}$ & $84.8^{\mathrm{a}(\mathrm{a})}$ & $60.0^{\mathrm{b}(\mathrm{b})}$ & 12.3 & $26.7^{\mathrm{a}(\mathrm{a})}$ & $31.3^{\mathrm{a}(\mathrm{a})}$ & $29.9^{\mathrm{a}(\mathrm{a})}$ & 7.3 \\
\hline Mean & & 94.5 & 89.9 & 70.0 & & 28.9 & 30.3 & 29.3 & \\
\hline$L S D$ & & 10.1 & 13.5 & 16.8 & & 11.1 & 11.0 & 6.5 & \\
\hline \multicolumn{10}{|l|}{$\mathrm{C} \times \mathrm{F}$} \\
\hline$\left(\mathrm{C} \times \mathrm{F}_{1}\right)$ & & $*$ & $*$ & $*$ & & ns & $*$ & $*$ & \\
\hline$\left(\mathrm{C} \times \mathrm{F}_{2}\right)$ & & $*$ & $*$ & ns & & $*$ & $\mathrm{~ns}$ & $*$ & \\
\hline$\left(\mathrm{C} \times \mathrm{F}_{3}\right)$ & & $*$ & $*$ & $\mathrm{~ns}$ & & $*$ & ns & ns & \\
\hline
\end{tabular}

Mean separation by LSD test. Mean values in the same column for each cultivar separately followed by different letters differ significantly at $p=0.05$. Means in each row for each seed characteristic separately followed by the same letter in parenthesis do not differ significantly at $p=0.05$.

$*=$ statistically significant $(p<0.05)$. 
Table 5. Effect of $\mathrm{N}$ levels on seed characteristics from pods harvested from different plant parts of four okra cultivars (Experiment 2)

\begin{tabular}{|c|c|c|c|c|c|c|c|c|c|}
\hline \multirow{2}{*}{ Cultivar (C) } & \multirow{2}{*}{$\begin{array}{l}\text { N level } \\
\left(\mathrm{mg} \mathrm{l}^{-1}\right)(\mathrm{F})\end{array}$} & \multicolumn{3}{|c|}{ Number of seeds/pod } & \multirow{2}{*}{ LSD } & \multicolumn{3}{|c|}{ 100-seed weight (g) } & \multirow{2}{*}{ LSD } \\
\hline & & Upper & Middle & Lower & & Upper & Middle & Lower & \\
\hline \multirow{3}{*}{ 'Boyiatiou' } & $150\left(\mathrm{~F}_{1}\right)$ & $70.7^{\mathrm{a}(\mathrm{a})}$ & $71.7^{\mathrm{a}(\mathrm{a})}$ & $65.5^{\mathrm{a}(\mathrm{a})}$ & 18.9 & $31.2^{\mathrm{ab}(\mathrm{a})}$ & $29.7^{\mathrm{a}(\mathrm{ab})}$ & $23.8^{\mathrm{a}(\mathrm{b})}$ & 6.1 \\
\hline & $300\left(\mathrm{~F}_{2}\right)$ & $70.0^{\mathrm{a}(\mathrm{a})}$ & $67.0^{\mathrm{ab}(\mathrm{a})}$ & $67.2^{\mathrm{a}(\mathrm{a})}$ & 5.6 & $30.3^{\mathrm{b}(\mathrm{a})}$ & $29.4^{\mathrm{a}(\mathrm{a})}$ & $28.6^{\mathrm{a}(\mathrm{a})}$ & 6.0 \\
\hline & $450\left(\mathrm{~F}_{3}\right)$ & $68.0^{\mathrm{a}(\mathrm{a})}$ & $59.0^{\mathrm{b}(\mathrm{a})}$ & $68.2^{\mathrm{a}(\mathrm{a})}$ & 9.7 & $35.7^{\mathrm{a}(\mathrm{a})}$ & $31.8^{\mathrm{a}(\mathrm{ab})}$ & $28.7^{\mathrm{a}(\mathrm{b})}$ & 5.2 \\
\hline Mean & & 69.5 & 65.9 & 66.9 & & 32.4 & 30.3 & 27.0 & \\
\hline \multirow[t]{2}{*}{$L S D$} & & 19.4 & 8.1 & 6.6 & & 5.4 & 5.7 & 6.2 & \\
\hline & $150\left(\mathrm{~F}_{1}\right)$ & $56.5^{\mathrm{b}(\mathrm{b})}$ & $73.5^{\mathrm{a}(\mathrm{a})}$ & $69.7^{\mathrm{a}(\mathrm{ab})}$ & 13.5 & $35.6^{\mathrm{a}(\mathrm{a})}$ & $42.0^{\mathrm{a}(\mathrm{a})}$ & $36.5^{\mathrm{a}(\mathrm{a})}$ & 8.6 \\
\hline \multirow[t]{2}{*}{ 'Veloudo’' } & $300\left(\mathrm{~F}_{2}\right)$ & $68.2^{\mathrm{ab}(\mathrm{b})}$ & $84.7^{\mathrm{a}(\mathrm{a})}$ & $61.0^{\mathrm{b}(\mathrm{b})}$ & 12.0 & $39.2^{\mathrm{a}(\mathrm{a})}$ & $37.5^{a(a)}$ & $37.9^{a(a)}$ & 8.9 \\
\hline & $450\left(\mathrm{~F}_{3}\right)$ & $75.0^{\mathrm{a}(\mathrm{a})}$ & $67.0^{\mathrm{b}(\mathrm{b})}$ & $67.2^{\mathrm{ab}(\mathrm{b})}$ & 6.9 & $38.3^{\mathrm{a}(\mathrm{a})}$ & $45.1^{\mathrm{a}(\mathrm{a})}$ & $40.5^{\mathrm{a}(\mathrm{a})}$ & 9.0 \\
\hline Mean & & 66.5 & 75.0 & 65.9 & & 37.7 & 41.5 & 38.3 & \\
\hline \multirow[t]{2}{*}{$L S D$} & & 16.4 & 8.4 & 6.1 & & 7.4 & 7.7 & 11.0 & \\
\hline & $150\left(\mathrm{~F}_{1}\right)$ & $70.0^{\mathrm{a}(\mathrm{a})}$ & $63.0^{\mathrm{b}(\mathrm{a})}$ & $75.0^{\mathrm{a}(\mathrm{a})}$ & 9.8 & $33.6^{\mathrm{a}(\mathrm{ab})}$ & $38.7^{a(a)}$ & $29.6^{\mathrm{a}(\mathrm{b})}$ & 8.5 \\
\hline \multirow[t]{2}{*}{ ‘Pylaias' } & $300\left(\mathrm{~F}_{2}\right)$ & $77.7^{\mathrm{a}(\mathrm{a})}$ & $72.5^{\mathrm{ab}(\mathrm{a})}$ & $73.5^{\mathrm{a}(\mathrm{a})}$ & 6.3 & $37.9^{\mathrm{a}(\mathrm{a})}$ & $36.5^{\mathrm{a}(\mathrm{a})}$ & $29.6^{\mathrm{a}(\mathrm{a})}$ & 8.8 \\
\hline & $450\left(\mathrm{~F}_{3}\right)$ & $66.5^{\mathrm{a}(\mathrm{a})}$ & $81.2^{\mathrm{a}(\mathrm{a})}$ & $82.5^{\mathrm{a}(\mathrm{a})}$ & 17.3 & $38.6^{\mathrm{a}(\mathrm{a})}$ & $32.2^{\mathrm{a}(\mathrm{ab})}$ & $25.7^{\mathrm{a}(\mathrm{b})}$ & 7.6 \\
\hline Mean & & 71.4 & 72.2 & 77.0 & & 36.7 & 35.8 & 28.3 & \\
\hline \multirow[t]{2}{*}{$L S D$} & & 9.3 & 10.5 & 15.4 & & 9.7 & 7.9 & 7.0 & \\
\hline & $150\left(\mathrm{~F}_{1}\right)$ & $86.5^{\mathrm{a}(\mathrm{b})}$ & $95.5^{\mathrm{a}(\mathrm{ab})}$ & $101.2^{\mathrm{a}(\mathrm{a})}$ & 11.5 & $31.0^{\mathrm{a}(\mathrm{a})}$ & $30.9^{\mathrm{a}(\mathrm{a})}$ & $26.6^{\mathrm{a}(\mathrm{a})}$ & 5.5 \\
\hline \multirow[t]{2}{*}{ 'Clemson' } & $300\left(\mathrm{~F}_{2}\right)$ & $88.5^{\mathrm{a}(\mathrm{a})}$ & $89.7^{\mathrm{a}(\mathrm{a})}$ & $86.7^{\mathrm{b}(\mathrm{a})}$ & 12.1 & $35.7^{\mathrm{a}(\mathrm{a})}$ & $32.2^{\mathrm{a}(\mathrm{ab})}$ & $27.7^{\mathrm{a}(\mathrm{b})}$ & 6.9 \\
\hline & $450\left(\mathrm{~F}_{3}\right)$ & $80.5^{\mathrm{a}(\mathrm{a})}$ & $85.2^{\mathrm{a}(\mathrm{a})}$ & $83.0^{\mathrm{c}(\mathrm{a})}$ & 17.1 & $35.9^{\mathrm{a}(\mathrm{a})}$ & $33.0^{\mathrm{a}(\mathrm{ab})}$ & $25.6^{\mathrm{a}(\mathrm{b})}$ & 7.9 \\
\hline Mean & & 85.1 & 90.1 & 90.3 & & 34.2 & 32.0 & 26.6 & \\
\hline$L S D$ & & 10.1 & 13.5 & 16.9 & & 7.2 & 6.8 & 6.5 & \\
\hline \multicolumn{10}{|l|}{$\mathrm{C} \times \mathrm{F}$} \\
\hline$\left(\mathrm{C} \times \mathrm{F}_{1}\right)$ & & $*$ & $*$ & $*$ & & ns & * & $*$ & \\
\hline$(\mathrm{C} \mathrm{x} \mathrm{F})$ & & $*$ & $*$ & $*$ & & * & * & $*$ & \\
\hline$\left(\mathrm{C} \times \mathrm{F}_{3}\right)$ & & $*$ & $*$ & $\mathrm{~ns}$ & & ns & $*$ & $*$ & \\
\hline
\end{tabular}

Mean separation by LSD test. Mean values in the same column followed by different letters differ significantly at $p=0.05 . *$ statistically significant $(p<0.05) ; \mathrm{ns}=$ not significant. (a): letter in parenthesis for horizontal analysis.

\subsection{0-Seed Weight}

Seed size expressed as the 100 seed weight was not affected by $\mathrm{N}$ level or the position of the pod on the plant in any cultivar in Experiment 1 (Table 4). Similarly, in Experiment 2 the 100 seed weight was not affected by N level, but was invariably lower in pods from the lower part of the plant compared with those from the middle and upper parts, but not always to a statistically significant degree (Table 5).

\subsection{Seed Moisture Content at Harvest and after Pod Drying}

The moisture content of seeds extracted from pods immediately after harvest was not significantly affected by the pod position on the plant or by the $\mathrm{N}$ level in either experiment and ranged from 25.5-37.5\% in Experiment 1 (Table 6) and 28.1-43.2\% in Experiment 2 (Table 8).

When the pods were dried at room temperature for 15 days and the seeds then extracted, the moisture content of seeds from the upper pods was higher at the higher $\mathrm{N}$ levels in 'Boyiatiou', 'Pylaias' and 'Clemson' (not in 'Veloudo') in Experiment 1 (Table 7), but not in Experiment 2 (Table 9). On the other hand, in Experiment 1 the moisture content of seeds from dried pods harvested from the middle and lower regions of the plant were mostly 
unaffected by $\mathrm{N}$ level (Table 7), whereas in Experiment 2 moisture content was higher at the higher $\mathrm{N}$ levels in 'Veloudo', 'Clemson' and 'Pylaias' (lower pods) and in 'Boyiatiou' and 'Clemson' (middle pods) (Table 9). Overall, there were no consistent differences in moisture content between seeds extracted from dried pods harvested from different regions of the plant (Tables 7, 9). During drying of the pods, seed moisture fell to 9.6-13.5\% (Experiment 1) and 11.0-12.9\% (Experiment 2).

Table 6. Effect of $\mathrm{N}$ levels on the moisture content and germination percentage of seeds extracted from the pods immediately after harvest in relation to plant part (Experiment 1)

\begin{tabular}{|c|c|c|c|c|c|c|c|c|c|}
\hline \multirow{3}{*}{ Cultivar (C) } & \multirow{3}{*}{$\begin{array}{l}\mathrm{N} \text { level } \\
\left(\mathrm{mg} \mathrm{l}^{-1}\right)(\mathrm{F})\end{array}$} & \multicolumn{8}{|c|}{ Seeds extracted from pods immediately after harvest } \\
\hline & & \multicolumn{3}{|c|}{ Moisture content at harvest (\%) } & \multirow{2}{*}{ LSD } & \multicolumn{3}{|c|}{ Germination $(\%)$} & \multirow{2}{*}{ LSD } \\
\hline & & Upper & Middle & Lower & & Upper & Middle & Lower & \\
\hline \multirow{3}{*}{ 'Boyiatiou' } & $150\left(\mathrm{~F}_{1}\right)$ & $29.9^{\mathrm{a}(\mathrm{ab})}$ & $18.9^{\mathrm{a}(\mathrm{b})}$ & $34.5^{a(a)}$ & 12.5 & $39.3^{\mathrm{a}(\mathrm{a})}$ & $43.4^{a(a)}$ & $44.2^{\mathrm{a}(\mathrm{a})}$ & 11.6 \\
\hline & $300\left(\mathrm{~F}_{2}\right)$ & $31.4^{\mathrm{a}(\mathrm{a})}$ & $29.9^{\mathrm{a}(\mathrm{a})}$ & $30.2^{\mathrm{a}(\mathrm{a})}$ & 15.0 & $44.8^{\mathrm{a}(\mathrm{a})}$ & $46.8^{\mathrm{a}(\mathrm{a})}$ & $44.4^{\mathrm{a}(\mathrm{a})}$ & 10.8 \\
\hline & $450\left(\mathrm{~F}_{3}\right)$ & $27.4^{\mathrm{a}(\mathrm{a})}$ & $27.8^{\mathrm{a}(\mathrm{a})}$ & $27.5^{\mathrm{a}(\mathrm{a})}$ & 14.2 & $47.6^{\mathrm{a}(\mathrm{a})}$ & $47.4^{\mathrm{a}(\mathrm{a})}$ & $46.7^{\mathrm{a}(\mathrm{a})}$ & 10.2 \\
\hline Mean & & 29.5 & 25.5 & 30.7 & & 43.9 & 45.8 & 45.1 & \\
\hline$L S D$ & & 14.8 & 13.5 & 13.5 & & 10.8 & 12.1 & 9.5 & \\
\hline \multirow{3}{*}{ 'Veloudo' } & $150\left(\mathrm{~F}_{1}\right)$ & $31.3^{\mathrm{a}(\mathrm{a})}$ & $27.5^{a(a)}$ & $29.3^{a(a)}$ & 13.4 & $47.6^{\mathrm{a}(\mathrm{b})}$ & $65.1^{\mathrm{b}(\mathrm{a})}$ & $56.0^{\mathrm{a}(\mathrm{ab})}$ & 13.2 \\
\hline & $300\left(\mathrm{~F}_{2}\right)$ & $30.9^{\mathrm{a}(\mathrm{a})}$ & $25.5^{\mathrm{a}(\mathrm{a})}$ & $32.0^{\mathrm{a}(\mathrm{a})}$ & 12.9 & $48.3^{\mathrm{a}(\mathrm{b})}$ & $78.2^{\mathrm{a}(\mathrm{a})}$ & $58.3^{\mathrm{a}(\mathrm{ab})}$ & 10.6 \\
\hline & $450\left(\mathrm{~F}_{3}\right)$ & $30.5^{\mathrm{a}(\mathrm{a})}$ & $30.3^{\mathrm{a}(\mathrm{a})}$ & $26.1^{\mathrm{a}(\mathrm{a})}$ & 13.8 & $52.9^{\mathrm{a}(\mathrm{b})}$ & $67.0^{\mathrm{ab}(\mathrm{a})}$ & $58.5^{\mathrm{a}(\mathrm{b})}$ & 8.6 \\
\hline Mean & & 30.9 & 27.7 & 29.1 & & 49.6 & 70.1 & 57.6 & \\
\hline$L S D$ & & 13.3 & 13.6 & 13.1 & & 9.8 & 12.3 & 10.6 & \\
\hline \multirow{3}{*}{ 'Pylaias' } & $150\left(\mathrm{~F}_{1}\right)$ & $35.7^{\mathrm{a}(\mathrm{a})}$ & $33.6^{\mathrm{a}(\mathrm{a})}$ & $35.7^{\mathrm{a}(\mathrm{a})}$ & 19.5 & $64.4^{\mathrm{a}(\mathrm{a})}$ & $65.9^{\mathrm{a}(\mathrm{a})}$ & $64.5^{\mathrm{a}(\mathrm{a})}$ & 11.1 \\
\hline & $300\left(\mathrm{~F}_{2}\right)$ & $39.4^{\mathrm{a}(\mathrm{a})}$ & $30.1^{a(a)}$ & $33.4^{a(a)}$ & 17.7 & $68.1^{\mathrm{a}(\mathrm{a})}$ & $68.4^{\mathrm{a}(\mathrm{a})}$ & $67.7^{\mathrm{a}(\mathrm{a})}$ & 11.9 \\
\hline & $450\left(\mathrm{~F}_{3}\right)$ & $37.6^{\mathrm{a}(\mathrm{a})}$ & $32.6^{a(a)}$ & $31.1^{\mathrm{a}(\mathrm{a})}$ & 17.1 & $77.1^{\mathrm{a}(\mathrm{a})}$ & $75.1^{\mathrm{a}(\mathrm{a})}$ & $73.2^{\mathrm{a}(\mathrm{a})}$ & 15.4 \\
\hline Mean & & 37.5 & 32.1 & 33.4 & & 69.8 & 69.8 & 68.4 & \\
\hline$L S D$ & & 18.4 & 17.2 & 18.7 & & 13.2 & 13.3 & 12.3 & \\
\hline \multirow{3}{*}{ 'Clemson' } & $150\left(\mathrm{~F}_{1}\right)$ & $33.3^{\mathrm{a}(\mathrm{a})}$ & $30.7^{7^{a(a)}}$ & $31.8^{\mathrm{a}(\mathrm{a})}$ & 17.1 & $75.0^{\mathrm{a}(\mathrm{a})}$ & $75.4^{\mathrm{a}(\mathrm{a})}$ & $73.8^{\mathrm{a}(\mathrm{a})}$ & 13.6 \\
\hline & $300\left(\mathrm{~F}_{2}\right)$ & $41.6^{\mathrm{a}(\mathrm{a})}$ & $30.9^{\mathrm{a}(\mathrm{a})}$ & $32.0^{\mathrm{a}(\mathrm{a})}$ & 18.1 & $75.9^{a(a)}$ & $74.2^{\mathrm{a}(\mathrm{a})}$ & $71.3^{\mathrm{a}(\mathrm{a})}$ & 13.2 \\
\hline & $450\left(\mathrm{~F}_{3}\right)$ & $35.3^{\mathrm{a}(\mathrm{a})}$ & $34.6^{a(a)}$ & $32.6^{\mathrm{a}(\mathrm{a})}$ & 16.4 & $78.1^{\mathrm{a}(\mathrm{a})}$ & $76.0^{\mathrm{a}(\mathrm{a})}$ & $78.1^{\mathrm{a}(\mathrm{a})}$ & 15.1 \\
\hline Mean & & 36.7 & 32.0 & 32.1 & & 76.3 & 75.2 & 74.4 & \\
\hline$L S D$ & & 16.9 & 17.3 & 17.5 & & 14.3 & 13.5 & 14.2 & \\
\hline \multicolumn{10}{|l|}{$\mathrm{C} \times \mathrm{F}$} \\
\hline$\left(\mathrm{C} \times \mathrm{F}_{1}\right)$ & & ns & ns & ns & & $*$ & $*$ & $*$ & \\
\hline$\left(\mathrm{C} \times \mathrm{F}_{2}\right)$ & & ns & ns & ns & & $*$ & $*$ & $*$ & \\
\hline$\left(\mathrm{C} \times \mathrm{F}_{3}\right)$ & & ns & ns & ns & & $*$ & $*$ & $*$ & \\
\hline
\end{tabular}

Mean separation by LSD test. Mean values in the same column followed by different letters differ significantly at $p=0.05 . *=$ statistically significant $(p<0.05)$; ns $=$ not significant. (a): letter in parenthesis for horizontal analysis. 
Table 7. Effect of $\mathrm{N}$ levels on the moisture content and germination percentage of seeds extracted from the pods 15 days after harvest in relation to plant part (Experiment 1)

\begin{tabular}{|c|c|c|c|c|c|c|c|c|c|}
\hline \multirow{3}{*}{ Cultivar (C) } & \multirow{3}{*}{$\begin{array}{l}\mathrm{N} \text { level } \\
\left(\mathrm{mg} \mathrm{l}^{-1}\right)(\mathrm{F})\end{array}$} & \multicolumn{8}{|c|}{ Seeds extracted from pods 15 days after harvest } \\
\hline & & \multicolumn{3}{|c|}{ Moisture content at harvest (\%) } & \multirow{2}{*}{ LSD } & \multicolumn{3}{|c|}{ Germination (\%) } & \multirow{2}{*}{ LSD } \\
\hline & & Upper & Middle & Lower & & Upper & Middle & Lower & \\
\hline \multirow{3}{*}{ 'Boyiatiou' } & $150\left(\mathrm{~F}_{1}\right)$ & $12.2^{\mathrm{b}(\mathrm{a})}$ & $13.2^{\mathrm{a}(\mathrm{a})}$ & $13.3^{\mathrm{a}(\mathrm{a})}$ & 1.5 & $46.3^{\mathrm{a}(\mathrm{c})}$ & $49.4^{\mathrm{b}(\mathrm{b})}$ & $54.0^{\mathrm{a}(\mathrm{a})}$ & 1.5 \\
\hline & $300\left(\mathrm{~F}_{2}\right)$ & $13.6^{\mathrm{ab}(\mathrm{ab})}$ & $12.6^{\mathrm{a}(\mathrm{b})}$ & $14.4^{\mathrm{a}(\mathrm{a})}$ & 1.0 & $51.3^{\mathrm{a}(\mathrm{b})}$ & $54.0^{\mathrm{ab}(\mathrm{a})}$ & $54.5^{\mathrm{a}(\mathrm{a})}$ & 1.0 \\
\hline & $450\left(\mathrm{~F}_{3}\right)$ & $14.0^{\mathrm{a}(\mathrm{a})}$ & $12.9^{\mathrm{a}(\mathrm{a})}$ & $12.8^{\mathrm{a}(\mathrm{a})}$ & 3.1 & $55.5^{\mathrm{a}(\mathrm{b})}$ & $62.2^{\mathrm{a}(\mathrm{a})}$ & $65.0^{\mathrm{a}(\mathrm{a})}$ & 3.1 \\
\hline Mean & & 13.2 & 12.9 & 13.5 & & 51.0 & 55.2 & 57.8 & \\
\hline$L S D$ & & 1.4 & 1.4 & 3.0 & & 8.9 & 10.2 & 12.1 & \\
\hline \multirow{3}{*}{ ‘Veloudo' } & $150\left(\mathrm{~F}_{1}\right)$ & $10.9^{\mathrm{b}(\mathrm{ab})}$ & $11.9^{\mathrm{a}(\mathrm{a})}$ & $8.8^{\mathrm{b}(\mathrm{b})}$ & 1.0 & $55.8^{\mathrm{a}(\mathrm{a})}$ & $61.2^{\mathrm{b}(\mathrm{a})}$ & $55.8^{\mathrm{b}(\mathrm{a})}$ & 1.0 \\
\hline & $300\left(\mathrm{~F}_{2}\right)$ & $9.8^{\mathrm{a}(\mathrm{b})}$ & $11.2^{\mathrm{a}(\mathrm{a})}$ & $9.4^{\mathrm{b}(\mathrm{b})}$ & 1.3 & $58.5^{\mathrm{a}(\mathrm{b})}$ & $63.8^{\mathrm{b}(\mathrm{a})}$ & $62.4^{\mathrm{ab}(\mathrm{a})}$ & 1.3 \\
\hline & $450\left(\mathrm{~F}_{3}\right)$ & $10.3^{\mathrm{b}(\mathrm{a})}$ & $9.1^{b(b)}$ & $10.8^{\mathrm{a}(\mathrm{a})}$ & 0.9 & $63.0^{\mathrm{a}(\mathrm{c})}$ & $72.1^{\mathrm{a}(\mathrm{a})}$ & $67.0^{\mathrm{a}(\mathrm{b})}$ & 0.9 \\
\hline Mean & & 10.3 & 10.7 & 9.6 & & 59.1 & 65.7 & 61.7 & \\
\hline$L S D$ & & 1.0 & 1.3 & 0.9 & & 9.5 & 8.6 & 9.7 & \\
\hline \multirow{3}{*}{ ‘Pylaias' } & $150\left(\mathrm{~F}_{1}\right)$ & $10.4^{\mathrm{b}(\mathrm{a})}$ & $9.5^{\mathrm{b}(\mathrm{a})}$ & $10.1^{\mathrm{a}(\mathrm{a})}$ & 1.0 & $71.9^{\mathrm{a}(\mathrm{ab})}$ & $71^{\mathrm{a}(\mathrm{b})}$ & $72.5^{\mathrm{a}(\mathrm{a})}$ & 1.0 \\
\hline & $300\left(\mathrm{~F}_{2}\right)$ & $10.6^{\mathrm{b}(\mathrm{a})}$ & $10.8^{\mathrm{a}(\mathrm{a})}$ & $10.9^{\mathrm{a}(\mathrm{a})}$ & 0.7 & $74.9^{\mathrm{a}(\mathrm{b})}$ & $78.5^{\mathrm{a}(\mathrm{a})}$ & $73.2^{\mathrm{a}(\mathrm{c})}$ & 0.7 \\
\hline & $450\left(\mathrm{~F}_{3}\right)$ & $12.1^{\mathrm{a}(\mathrm{a})}$ & $9.8^{\mathrm{b}(\mathrm{b})}$ & $11.0^{\mathrm{a}(\mathrm{ab})}$ & 1.2 & $80.4^{\mathrm{a}(\mathrm{a})}$ & $79.6^{\mathrm{a}(\mathrm{ab})}$ & $78.5^{\mathrm{a}(\mathrm{b}}$ & 1.2 \\
\hline Mean & & 11.0 & 10.0 & 10.6 & & 75.7 & 76.3 & 74.7 & \\
\hline$L S D$ & & 1.0 & 0.9 & 1.1 & & 14.3 & 14.5 & 14.4 & \\
\hline \multirow{3}{*}{ 'Clemson' } & $150\left(\mathrm{~F}_{1}\right)$ & $9.7^{\mathrm{b}(\mathrm{a})}$ & $9.8^{\mathrm{b}(\mathrm{a})}$ & $10.4^{\mathrm{a}(\mathrm{a})}$ & 0.9 & $82.3^{\mathrm{a}(\mathrm{a})}$ & $78.3^{\mathrm{a}(\mathrm{a})}$ & $79.8^{\mathrm{a}(\mathrm{a})}$ & 14.6 \\
\hline & $300\left(F_{2}\right)$ & $11.2^{\mathrm{a}(\mathrm{a})}$ & $11.7^{\mathrm{a}(\mathrm{a})}$ & $10.4^{\mathrm{a}(\mathrm{a})}$ & 1.2 & $81.4^{\mathrm{a}(\mathrm{a})}$ & $79.2^{\mathrm{a}(\mathrm{a})}$ & $76.1^{\mathrm{a}(\mathrm{a})}$ & 14.1 \\
\hline & $450\left(\mathrm{~F}_{3}\right)$ & $10.4^{\mathrm{a}(\mathrm{a})}$ & $10.7^{\mathrm{a}(\mathrm{a})}$ & $10.2^{\mathrm{a}(\mathrm{a})}$ & 1.2 & $80.9^{\mathrm{a}(\mathrm{a})}$ & $81.7^{\mathrm{a}(\mathrm{a})}$ & $81.5^{\mathrm{a}(\mathrm{a})}$ & 15.2 \\
\hline Mean & & 10.4 & 10.7 & 10.3 & & 81.5 & 79.7 & 79.1 & \\
\hline$L S D$ & & 1.0 & 1.1 & 1.2 & & 15.0 & 14.4 & 14.5 & \\
\hline \multicolumn{10}{|l|}{$\mathrm{C} \times \mathrm{F}$} \\
\hline$\left(\mathrm{C} \times \mathrm{F}_{1}\right)$ & & * & $*$ & $*$ & & $*$ & * & * & \\
\hline$\left(\mathrm{C} \times \mathrm{F}_{2}\right)$ & & $*$ & $*$ & $*$ & & $*$ & $*$ & $*$ & \\
\hline$\left(\mathrm{C} \times \mathrm{F}_{3}\right)$ & & $*$ & $*$ & ns & & $*$ & * & $*$ & \\
\hline
\end{tabular}

Mean separation by LSD test. Mean values in the same column followed by different letters differ significantly at $p=0.05 . *=$ statistically significant $(p<0.05) ; \mathrm{ns}=$ not significant. (a): letter in parenthesis for horizontal analysis.

\subsection{Seed Germination}

In Experiment 1, germination was not affected by $\mathrm{N}$ level when seeds were extracted from freshly harvested pods (i.e. without drying) and varied between 43.9 and $76.3 \%$, being highest in 'Clemson' and lowest in 'Boyiatiou' irrespective of $\mathrm{N}$ level or the position of the pods on the plant (Table 6). A similar result was recorded in Experiment 2, where the germination ranged from $48.1 \%$ ('Boyiatiou') to $75.6 \%$ ('Clemson') (Table 8). With the exception of 'Veloudo' in Experiment 1 (where germination was higher for seeds from the pods in the middle compared to the lower region of the plant), no differences in germination were recorded in relation to the position of the pods on the plant (Tables 6 and 8). 
Table 8. Effect of $\mathrm{N}$ levels on the moisture content and germination percentage of seeds extracted from the pods immediately after harvest in relation to plant part (Experiment 2)

\begin{tabular}{|c|c|c|c|c|c|c|c|c|c|}
\hline \multirow{3}{*}{ Cultivar (C) } & \multirow{3}{*}{$\begin{array}{l}\text { N level } \\
\left(\mathrm{mg} \mathrm{l}^{-1}\right)(\mathrm{F})\end{array}$} & \multicolumn{8}{|c|}{ Seeds extracted from pods immediately after harvest } \\
\hline & & \multicolumn{3}{|c|}{ Moisture content at harvest (\%) } & \multirow{2}{*}{ LSD } & \multicolumn{3}{|c|}{ Germination (\%) } & \multirow{2}{*}{ LSD } \\
\hline & & Upper & Middle & Lower & & Upper & Middle & Lower & \\
\hline \multirow{3}{*}{ 'Boyiatiou' } & $150\left(\mathrm{~F}_{1}\right)$ & $31.1^{\mathrm{a}(\mathrm{a})}$ & $31.0^{\mathrm{a}(\mathrm{a})}$ & $33.6^{\mathrm{a}(\mathrm{a})}$ & 13.0 & $47.2^{\mathrm{a}(\mathrm{a})}$ & $47.1^{\mathrm{b}(\mathrm{a})}$ & $48.7^{\mathrm{a}(\mathrm{a})}$ & 8.6 \\
\hline & $300\left(\mathrm{~F}_{2}\right)$ & $31.0^{\mathrm{a}(\mathrm{a})}$ & $22.6^{\mathrm{a}(\mathrm{a})}$ & $30.8^{\mathrm{a}(\mathrm{a})}$ & 12.5 & $48.0^{\mathrm{a}(\mathrm{a})}$ & $51.2^{\mathrm{ab}(\mathrm{a})}$ & $49.0^{\mathrm{a}(\mathrm{a})}$ & 8.8 \\
\hline & $450\left(\mathrm{~F}_{3}\right)$ & $35.5^{\mathrm{a}(\mathrm{a})}$ & $30.7^{\mathrm{a}(\mathrm{a})}$ & $30.1^{\mathrm{a}(\mathrm{a})}$ & 13.3 & $49.2^{\mathrm{a}(\mathrm{a})}$ & $57.2^{\mathrm{a}(\mathrm{a})}$ & $55.5^{\mathrm{a}(\mathrm{a})}$ & 10.9 \\
\hline Mean & & 32.5 & 28.1 & 31.5 & & 48.1 & 51.8 & 51.0 & \\
\hline$L S D$ & & 13.5 & 13.0 & 12.4 & & 8.5 & 9.5 & 10.4 & \\
\hline \multirow{3}{*}{ 'Veloudo' } & $150\left(\mathrm{~F}_{1}\right)$ & $31.0^{\mathrm{a}(\mathrm{a})}$ & $30.6^{\mathrm{a}(\mathrm{a})}$ & $38.9^{\mathrm{a}(\mathrm{a})}$ & 13.5 & $51.9^{\mathrm{a}(\mathrm{a})}$ & $54.6^{\mathrm{b}(\mathrm{a})}$ & $56.6^{\mathrm{a}(\mathrm{a})}$ & 9.2 \\
\hline & $300\left(F_{2}\right)$ & $32.5^{\mathrm{a}(\mathrm{a})}$ & $24.5^{\mathrm{a}(\mathrm{a})}$ & $29.1^{\mathrm{a}(\mathrm{a})}$ & 13.0 & $53.9^{\mathrm{a}(\mathrm{a})}$ & $57.5^{\mathrm{ab}(\mathrm{a})}$ & $59.8^{\mathrm{a}(\mathrm{a})}$ & 10.5 \\
\hline & $450\left(\mathrm{~F}_{3}\right)$ & $33.5^{\mathrm{a}(\mathrm{a})}$ & $30.6^{\mathrm{a}(\mathrm{a})}$ & $29.4^{\mathrm{a}(\mathrm{a})}$ & 12.4 & $58.9^{\mathrm{a}(\mathrm{a})}$ & $65.0^{\mathrm{a}(\mathrm{a})}$ & $61.7^{\mathrm{a}(\mathrm{a})}$ & 8.9 \\
\hline Mean & & 32.3 & 28.5 & 32.4 & & 54.9 & 59.0 & 59.3 & \\
\hline$L S D$ & & 13.0 & 12.4 & 11.2 & & 10.1 & 8.7 & 9.8 & \\
\hline \multirow{3}{*}{ 'Pylaias' } & $150\left(\mathrm{~F}_{1}\right)$ & $33.7^{\mathrm{a}(\mathrm{a})}$ & $43.7^{\mathrm{a}(\mathrm{a})}$ & $35.7^{\mathrm{a}(\mathrm{a})}$ & 15.0 & $67.4^{\mathrm{a}(\mathrm{a})}$ & $70.7^{\mathrm{a}(\mathrm{a})}$ & $69.6^{\mathrm{a}(\mathrm{a})}$ & 11.6 \\
\hline & $300\left(\mathrm{~F}_{2}\right)$ & $33.0^{\mathrm{a}(\mathrm{a})}$ & $43.5^{\mathrm{a}(\mathrm{a})}$ & $31.5^{\mathrm{a}(\mathrm{a})}$ & 14.9 & $71.1^{\mathrm{a}(\mathrm{a})}$ & $72.3^{\mathrm{a}(\mathrm{a})}$ & $70.4^{\mathrm{a}(\mathrm{a})}$ & 13.1 \\
\hline & $450\left(\mathrm{~F}_{3}\right)$ & $33.0^{\mathrm{a}(\mathrm{a})}$ & $42.5^{\mathrm{a}(\mathrm{a})}$ & $32.9^{\mathrm{a}(\mathrm{a})}$ & 14.7 & $72.3^{\mathrm{a}(\mathrm{a})}$ & $74.0^{\mathrm{a}(\mathrm{a})}$ & $69.9^{\mathrm{a}(\mathrm{a})}$ & 14.5 \\
\hline Mean & & 33.2 & 43.2 & 33.3 & & 70.2 & 72.3 & 69.9 & \\
\hline$L S D$ & & 16.7 & 13.6 & 14.2 & & 13.8 & 12.9 & 12.5 & \\
\hline \multirow{3}{*}{ 'Clemson' } & $150\left(\mathrm{~F}_{1}\right)$ & $40.2^{\mathrm{a}(\mathrm{a})}$ & $32.8^{\mathrm{a}(\mathrm{a})}$ & $26.3^{\mathrm{a}(\mathrm{a})}$ & 14.9 & $67.9^{\mathrm{a}(\mathrm{a})}$ & $72.6^{\mathrm{a}(\mathrm{a})}$ & $66.9^{\mathrm{a}(\mathrm{a})}$ & 16.2 \\
\hline & $300\left(F_{2}\right)$ & $39.7^{\mathrm{a}(\mathrm{a})}$ & $40.4^{\mathrm{a}(\mathrm{a})}$ & $33.5^{\mathrm{a}(\mathrm{a})}$ & 11.8 & $71.5^{\mathrm{a}(\mathrm{a})}$ & $76.0^{\mathrm{a}(\mathrm{a})}$ & $70.2^{\mathrm{a}(\mathrm{a})}$ & 17.7 \\
\hline & $450\left(\mathrm{~F}_{3}\right)$ & $38.4^{\mathrm{a}(\mathrm{a})}$ & $29.0^{\mathrm{a}(\mathrm{a})}$ & $29.5^{\mathrm{a}(\mathrm{a})}$ & 14.3 & $71.1^{\mathrm{a}(\mathrm{a})}$ & $78.3^{\mathrm{a}(\mathrm{a})}$ & $74.3^{\mathrm{a}(\mathrm{a})}$ & 16.5 \\
\hline Mean & & 39.4 & 34.0 & 29.7 & & 70.1 & 75.6 & 70.4 & \\
\hline$L S D$ & & 13.8 & 13.1 & 14.3 & & 16.3 & 16.0 & 18.0 & \\
\hline \multicolumn{10}{|l|}{$\mathrm{CxF}$} \\
\hline$\left(\mathrm{C} \times \mathrm{F}_{1}\right)$ & & ns & ns & $*$ & & $*$ & $*$ & $*$ & \\
\hline$\left(\mathrm{C} \times \mathrm{F}_{2}\right)$ & & $\mathrm{ns}$ & $*$ & $\mathrm{~ns}$ & & $*$ & $*$ & $*$ & \\
\hline$\left(\mathrm{C} \times \mathrm{F}_{3}\right)$ & & $\mathrm{ns}$ & $*$ & $\mathrm{~ns}$ & & $*$ & $*$ & $*$ & \\
\hline
\end{tabular}

Mean separation by LSD test. Mean values in the same column followed by different letters differ significantly at $p=0.05 . *=$ statistically significant $(p<0.05) ; \mathrm{ns}=$ not significant. (a): letter in parenthesis for horizontal analysis. 
Table 9. Effect of $\mathrm{N}$ levels on the moisture content and germination percentage of seeds extracted from the pods 15 days after harvest in relation to plant part (Experiment 2)

\begin{tabular}{|c|c|c|c|c|c|c|c|c|c|}
\hline \multirow{3}{*}{ Cultivar (C) } & \multirow{3}{*}{$\begin{array}{l}\text { N level } \\
\left(\mathrm{mg} \mathrm{l}^{-1}\right)(\mathrm{F})\end{array}$} & \multicolumn{8}{|c|}{ Seeds extracted from pods 15 days after harvest } \\
\hline & & \multicolumn{3}{|c|}{ Moisture content at harvest $(\%)$} & \multirow{2}{*}{ LSD } & \multicolumn{3}{|c|}{ Germination $(\%)$} & \multirow{2}{*}{ LSD } \\
\hline & & Upper & Middle & Lower & & Upper & Middle & Lower & \\
\hline \multirow{3}{*}{ 'Boyiatiou' } & $150\left(\mathrm{~F}_{1}\right)$ & $12.8^{\mathrm{a}(\mathrm{a})}$ & $11.4^{\mathrm{b}(\mathrm{a})}$ & $11.4^{\mathrm{a}(\mathrm{a})}$ & 1.3 & $47.5^{\mathrm{b}(\mathrm{a})}$ & $54.5^{\mathrm{b}(\mathrm{a})}$ & $54.5^{\mathrm{a}(\mathrm{a})}$ & 9.2 \\
\hline & $300\left(\mathrm{~F}_{2}\right)$ & $12.9^{\mathrm{a}(\mathrm{a})}$ & $11.6^{\mathrm{b}(\mathrm{b})}$ & $12.0^{\mathrm{a}(\mathrm{ab})}$ & 1.0 & $52.2^{\mathrm{ab}(\mathrm{a})}$ & $57.4^{\mathrm{ab}(\mathrm{a})}$ & $59.3^{\mathrm{a}(\mathrm{a})}$ & 10.4 \\
\hline & $450\left(\mathrm{~F}_{3}\right)$ & $13.2^{\mathrm{a}(\mathrm{b})}$ & $15.8^{\mathrm{a}(\mathrm{a})}$ & $12.6^{\mathrm{a}(\mathrm{b})}$ & 2.5 & $57.5^{\mathrm{a}(\mathrm{a})}$ & $65.6^{\mathrm{a}(\mathrm{a})}$ & $63.3^{\mathrm{a}(\mathrm{a})}$ & 11.1 \\
\hline Mean & & 12.9 & 12.9 & 12.0 & & 52.4 & 59.1 & 59.0 & \\
\hline$L S D$ & & 0.9 & 2.4 & 1.4 & & 9.5 & 9.9 & 11.3 & \\
\hline \multirow{3}{*}{ 'Veloudo' } & $150\left(\mathrm{~F}_{1}\right)$ & $11.5^{\mathrm{a}(\mathrm{ab})}$ & $12.3^{\mathrm{a}(\mathrm{a})}$ & $10.9^{\mathrm{b}(\mathrm{b})}$ & 1.2 & $58.5^{\mathrm{b}(\mathrm{a})}$ & $62.1^{\mathrm{b}(\mathrm{a})}$ & $60.9^{\mathrm{a}(\mathrm{a})}$ & 9.3 \\
\hline & $300\left(\mathrm{~F}_{2}\right)$ & $11.2^{\mathrm{a}(\mathrm{a})}$ & $9.7^{\mathrm{b}(\mathrm{b})}$ & $12.4^{\mathrm{a}(\mathrm{a})}$ & 1.2 & $62.8^{\mathrm{ab}(\mathrm{a})}$ & $66.9^{\mathrm{ab}(\mathrm{a})}$ & $64.3^{\mathrm{a}(\mathrm{a})}$ & 10.3 \\
\hline & $450\left(\mathrm{~F}_{3}\right)$ & $10.5^{\mathrm{a}(\mathrm{b})}$ & $11.8^{\mathrm{a}(\mathrm{ab})}$ & $13.0^{\mathrm{a}(\mathrm{a})}$ & 1.4 & $68.0^{\mathrm{a}(\mathrm{a})}$ & $72.9^{\mathrm{a}(\mathrm{a})}$ & $69.9^{\mathrm{a}(\mathrm{a})}$ & 10.1 \\
\hline Mean & & 11.0 & 11.2 & 12.1 & & 63.1 & 67.3 & 65.0 & \\
\hline$L S D$ & & 1.3 & 0.8 & 1.6 & & 9.0 & 10.0 & 10.7 & \\
\hline \multirow{3}{*}{ 'Pylaias' } & $150\left(\mathrm{~F}_{1}\right)$ & $11.4^{\mathrm{b}(\mathrm{a})}$ & $12.1^{\mathrm{a}(\mathrm{a})}$ & $11.1^{\mathrm{b}(\mathrm{a})}$ & 0.8 & $70.0^{\mathrm{a}(\mathrm{a})}$ & $71.8^{\mathrm{a}(\mathrm{a})}$ & $73.5^{\mathrm{a}(\mathrm{a})}$ & 11.8 \\
\hline & $300\left(\mathrm{~F}_{2}\right)$ & $14.9^{\mathrm{a}(\mathrm{a})}$ & $12.1^{\mathrm{a}(\mathrm{b})}$ & $11.7^{\mathrm{b}(\mathrm{b})}$ & 2.3 & $72.7^{\mathrm{a}(\mathrm{a})}$ & $76.0^{\mathrm{a}(\mathrm{a})}$ & $68.0^{\mathrm{a}(\mathrm{a})}$ & 15.8 \\
\hline & $450\left(\mathrm{~F}_{3}\right)$ & $10.6^{\mathrm{b}(\mathrm{b})}$ & $11.1^{\mathrm{b}(\mathrm{b})}$ & $12.4^{\mathrm{a}(\mathrm{a})}$ & 0.6 & $74.6^{\mathrm{a}(\mathrm{a})}$ & $75.5^{\mathrm{a}(\mathrm{a})}$ & $73.1^{\mathrm{a}(\mathrm{a})}$ & 15.0 \\
\hline Mean & & 12.3 & 11.7 & 11.7 & & 72.4 & 74.4 & 71.5 & \\
\hline$L S D$ & & 2.2 & 0.7 & 0.9 & & 13.4 & 14.4 & 15.1 & \\
\hline \multirow{3}{*}{ 'Clemson' } & $150\left(\mathrm{~F}_{1}\right)$ & $11.2^{\mathrm{a}(\mathrm{a})}$ & $11.9^{\mathrm{b}(\mathrm{a})}$ & $10.0^{\mathrm{b}(\mathrm{b})}$ & 0.9 & $73.5^{\mathrm{a}(\mathrm{a})}$ & $75.1^{\mathrm{a}(\mathrm{a})}$ & $71.3^{\mathrm{a}(\mathrm{a})}$ & 16.7 \\
\hline & $300\left(F_{2}\right)$ & $11.9^{\mathrm{a}(\mathrm{a})}$ & $11.7^{\mathrm{b}(\mathrm{a})}$ & $11.8^{\mathrm{a}(\mathrm{a})}$ & 1.4 & $74.5^{\mathrm{a}(\mathrm{a})}$ & $78.2^{\mathrm{a}(\mathrm{a})}$ & $73.0^{\mathrm{a}(\mathrm{a})}$ & 17.6 \\
\hline & $450\left(\mathrm{~F}_{3}\right)$ & $12.0^{\mathrm{a}(\mathrm{b})}$ & $13.2^{\mathrm{a}(\mathrm{a})}$ & $11.6^{\mathrm{a}(\mathrm{b})}$ & 1.0 & $75.3^{\mathrm{a}(\mathrm{a})}$ & $79.7^{\mathrm{a}(\mathrm{a})}$ & $77.1^{\mathrm{a}(\mathrm{a})}$ & 16.8 \\
\hline Mean & & 11.7 & 12.2 & 11.1 & & 74.4 & 77.6 & 73.8 & \\
\hline$L S D$ & & 1.3 & 1.1 & 0.9 & & 15.8 & 16.3 & 18.9 & \\
\hline \multicolumn{10}{|l|}{$\mathrm{C} \times \mathrm{F}$} \\
\hline$\left(\mathrm{C} \times \mathrm{F}_{1}\right)$ & & $*$ & ns & $*$ & & $*$ & $*$ & $*$ & \\
\hline$\left(\mathrm{C} \times \mathrm{F}_{2}\right)$ & & $*$ & $*$ & ns & & $*$ & $*$ & $\mathrm{~ns}$ & \\
\hline$\left(\mathrm{C} \times \mathrm{F}_{3}\right)$ & & $*$ & $*$ & $*$ & & $*$ & $*$ & $\mathrm{~ns}$ & \\
\hline
\end{tabular}

Mean separation by LSD test. Mean values in the same column followed by different letters differ significantly at $p=0.05$. $^{*}=$ statistically significant $(p<0.05)$; ns $=$ not significant. (a): letter in parenthesis for horizontal analysis.

When pods were dried prior to seed extraction, germination was not affected by $\mathrm{N}$ in seeds from the upper and lower pods in Experiment 1, but in 'Boyiatiou' and 'Veloudo' was higher for seeds from pods in the middle region of the plant from the $450 \mathrm{ppm} \mathrm{N}$ treatment than the $150 \mathrm{ppm} \mathrm{N}$ treatment (Table 7). In Experiment 2, a similar result was recorded, with differences in germination being recorded only for 'Boyiatiou' and 'Veloudo' (higher germination in seeds from $450 \mathrm{ppm} \mathrm{N}$ compared to $150 \mathrm{ppm} \mathrm{N}$ for pods harvested from the upper and middle part $\mathrm{s}$ of the plant (Table 9). In Experiment 1, germination was higher in seeds from the lower pods compared to the upper pods for 'Boyiatiou' (at all $\mathrm{N}$ levels) and 'Veloudo' (450 ppm N), but lower in 'Pylaias' (300 ppm N) (Table 7). In Experiment 2, no effect of pod position was observed in relation to the germination of seeds from dried pods (Table 9).

\subsection{Germination of Seeds in Relation to Storage}

In both experiments, the percent germination of seeds of all cultivars was higher after storage for 18 months at room temperature compared with the initial germination, irrespective of whether seeds were extracted from pods 
immediately or 15 days after harvest (Figures 4 and 5). It is noteworthy that whereas in both experiments the germination of 'Boyiatiou' and 'Veloudo' was initially $<65 \%$ (i.e. lower than the minimum acceptable level, FEK 140/2000) (the official standard for commercial purpose is $85 \%$ ), following storage it reached $75-85 \%$ ('Boyiatiou') and 85-90\% ('Veloudo').

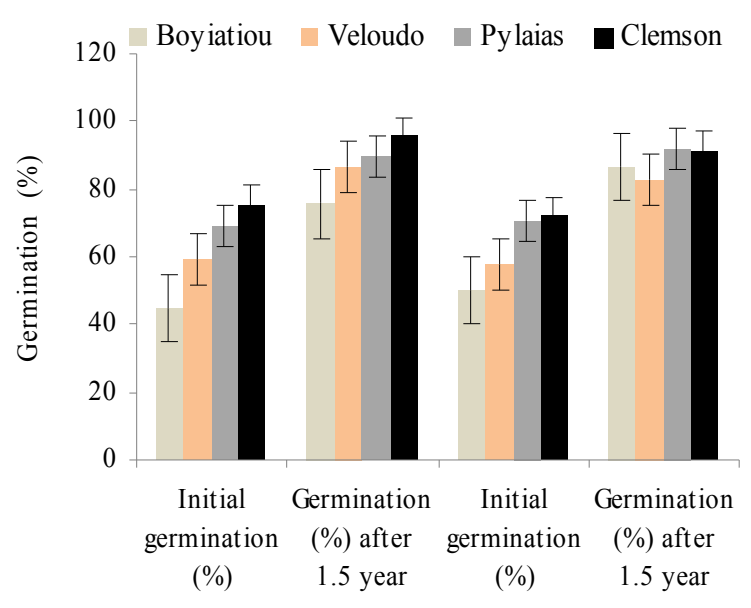

Experiment 1

Exp eriment 2

Figure 4. The germination percentage of seeds of four okra cultivars extracted from pods immediately after harvest in Experiments 1 and 2: (a) Initial germination, (b) After storage for 18 months

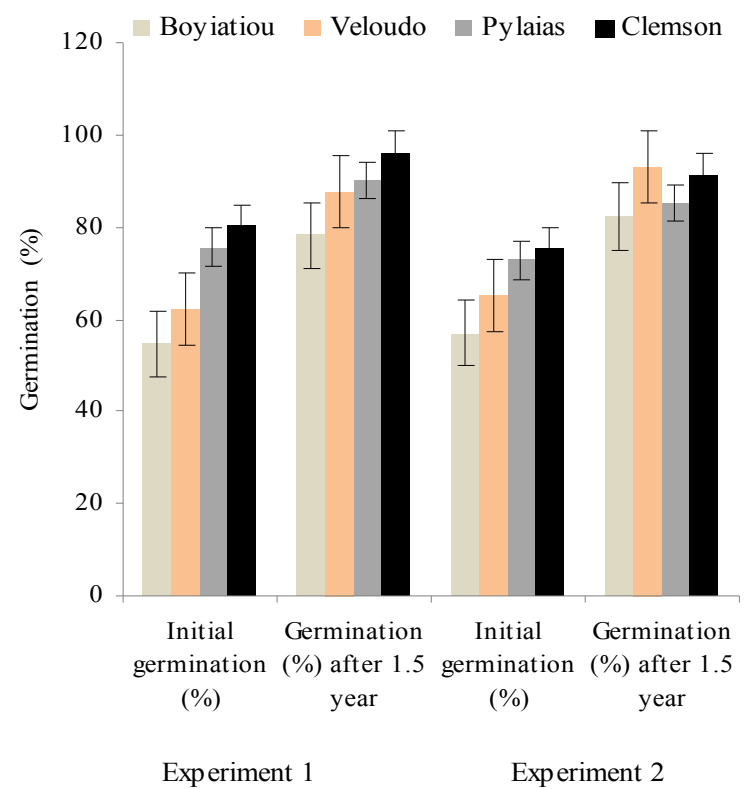

Figure 5. The percent germination of seeds of four okra cultivars extracted from pods 15 days after harvest in

Experiments 1 and 2: (a) Initial germination, (b) After storage for 18 months

\section{Discussion}

The beneficial effects of $\mathrm{N}$ application on okra growth and pod production are well documented, especially for fresh production (Prabhakar, Hebbar, \& Gayathri, 2009) and to a lesser extent for seed crops (Dutta \& Naik, 2009). There is, however, little available information in the literature concerning the effect of $\mathrm{N}$ on pod characteristics and seed quality.

From the results of the present experiments, it is clear that although significant differences in flower induction 
and pod set existed between cultivars, overall there was little or no effect of $\mathrm{N}$ application on these two characteristics. In both experiments, flower induction was highest in 'Clemson' and lowest in 'Boyiatiou' and although pod set increased at $450 \mathrm{ppm} \mathrm{N}$ application in 'Boyiatiou', this did not occur in the other cultivars. It should be noted, however, that pod set in 'Boyiatiou' was much lower than that in the other cultivars: for example mean pod set was 7.3 and 6.3 pods per plant in 'Boyiatiou' compared with 24.3 and 21.2 pods per plant in 'Clemson' (Experiments 1 and 2, respectively). This result is somewhat surprising since 'Boyiatiou' is a local cultivar (Koutsos, 2009) and might be expected to be better adapted to Greek climatic conditions, e.g. light, temperature and humidity, than the American cv. 'Clemson'. Although okra is considered to be a short-day plant (Bhatt \& Srinivasa Rao, 2009), cv. 'Boyiatiou' (like 'Pylaias') is classified as day-neutral (Koutsos, 2009). Similarly, although pod length and diameter varied between the cultivars, these characteristics were largely unaffected by N level, despite reports to the contrary by Majanbu et al. (1985) and Singh (1995), irrespective of the position of the pods on the plant, presumably because pod dimensions are genetically determined rather than influenced by cultivation conditions (Raji, 1994).

The number of seeds per pod was significantly higher in the pods from the middle and upper part of the plant in 'Clemson' and 'Pylaias', as well as in 'Boyiatiou' (at $450 \mathrm{ppm} \mathrm{N}$ ) and 'Veloudo' (at $150 \mathrm{ppm} \mathrm{N}$ ) in Experiment 1 (Table 4), but only in 'Veloudo' (350 and $450 \mathrm{ppm} \mathrm{N}$ ) in Experiment 2. Although Majanbu et al. (1985) and Moniruzzaman and Quamruzzaman (2009) reported a positive effect of $\mathrm{N}$ on the number of seeds per fruit, in our experiments the $\mathrm{N}$ level had virtually no effect on seed number in any cultivar in both experiments. It is possible that these differences relate to the cultivars used in each study, or to differences in climatic conditions. Moreover, these authors did not study the effect of pod position on seed number, which the data of Table 4 show to be a significant factor in defining seed number per pod.

No significant effect of $\mathrm{N}$ on seed size (100-seed weight) was detected, irrespective of the pod position at harvest (upper, middle and lower) or cultivar. This result contrasts with those of Uddin, Mondal, Samsuzzaman, and Siddique (2006) and Moniruzzaman and Quamruzzaman (2009) who reported a positive effect of N on mean 100 seed weight. The percent germination of seeds extracted immediately after harvest or after storage of pods for 15 days before seed extraction was not affected by $\mathrm{N}$ application; nor was seed moisture content. The differences in germination ability between cultivars appear to relate to variation of seed hardness at harvest since pods were harvested at a mature stage. As noted elsewhere, 'Boyiatiou' is especially susceptible to seed hardness, which accounts for its lower percent germination (49.7\%) in comparison with 'Clemson' (77.7\%) (Passam \& Rekoumi, 2009).

It is interesting that the only consistently positive effects of $\mathrm{N}$ application on seed germination were detected in 'Boyiatiou'. Although frequently insignificant, an increase in germination was nevertheless observed irrespective of the position of the pod on the plant. The increase of germination was relatively higher in the case of seeds extracted from pods after storage for 15 days at room temperature, i.e. in seeds with a lower moisture content than those extracted immediately after harvest. Seed hardness in okra is known to relate to moisture content and the rate of seed drying (Demir, 1997), whereas in other species $\mathrm{N}$ delays flowering and fruit maturation (George, 1987). It may be that in 'Boyiatiou', high $\mathrm{N}$ levels delay the rate of seed maturation and thereby reduce the incidence of hard seeds. It is surprising, however, that the germination of 'Boyiatiou' was not affected by the method of seed extraction since the higher moisture content of seeds extracted from pods immediately after harvest might have been expected to lead to higher germination compared with seeds from dried pods (mean moisture content $25-32 \%$ and $12-13 \%$, respectively). Perhaps, the drying of pods prior to seed extraction permitted after-ripening of seeds, as occurs in aubergine (Solanum melongena L.) (Passam, Theodoropoulou, Karanissa, \& Karapanos, 2010).

The effect of pod position on seed quality in okra has already been reported by Purquerio, Lago, and Passos (2010). Yadav et al. (2001) observed higher seed germination from pods harvested from lower positions on the okra plant, while Prabhakar, Hedge, Srinivas, and Doijode (1985) and Naik, Vogeesha, Bhanuprakash \& Padmini (2004) reported that seeds harvested from okra pods produced at the lower nodes (up to the $8^{\text {th }}$ node) had better quality. Overall, our data do not support these reports (with a few exceptions, Tables 6 and 7), indicating that under the hot and dry conditions of Thessaly during the summer, the rate of seed drying is similar (and therefore the occurrence of hard seeds) for pods, irrespective of their position on the plant.

Seed germination in all the cultivars increased significantly after storage for 18 months, irrespective of the method of seed extraction. Mohammadi, Khah, and Bannayan (2011) reported a similar positive effect of seed storage for 12 months on okra germination. In this way, seed lots of 'Boyiatiou' that were initially below the minimum requirement for germination (FEK 140/2000), attained an acceptable level of germination after storage, according to Greek market standards for seed trading. 


\section{Conclusions}

The present experiments provide new information on the seed quality of four cultivars of okra in relation to $\mathrm{N}$ level and the part (upper, lower and middle) of the plant from which harvest took place. $\mathrm{N}$ application generally increased flowering, but pod set increased only in 'Boyiatiou'. Pod dimensions (length and diameter) were largely unaffected by $\mathrm{N}$ level, irrespective of the position of the pods on the plant, as was the 100-seed weight and seed germination. The slight (insignificant) increase in seed germination of 'Boyiatiou' in response to $\mathrm{N}$ is worthy of further study in view of the susceptibility of this cultivar to seed hardness. There was no benefit of pod after-ripening, but seed storage for 18 months at room temperature proved to be a positive way to improve seed quality, especially in 'Boyiatiou'.

\section{Acknowledgements}

We are grateful to the National Foundation of Scholarships of Greece for its financial support to Mohammadi Ghadir throughout the course of this work.

\section{References}

Amjad, M., Anjum, M. A., \& Hussain, S. (2001). Effect of different sowing dates and various doses of fertilizer on juvenility and productivity of okra. Pakistan Journal of Agriculturanl Science, 38, 29-32.

Anjum, M. A., \& Amjad, M. (1999). Response of okra (Abelmoschus esculentus L. Moench) to different levels of $\mathrm{N}, \mathrm{P}$ and $\mathrm{K}$ fertilizers. Pakistan Journal of BioIogical Sciences, 2, 794-796. http://dx.doi.org/10.3923/pjbs.1999.794.796

Asif, M. T., \& Greig, J. K. (1972). Effects of N, P and K fertilization on fruit yield, macro- and micro-nutrient levels and nitrate accumulation in okra (Abelmoschus esculentus L. Moench). Journal of the American Society for Horticultural Science, 97, 440-442.

Auld, D. L., Bettis, B. L., Crock, J. E., \& Kephart, D. (1988). Planting date and temperature effects on germination, and seed yield of chickpea. Agronomy Journal, 80, 909-914. http://dx.doi.org/10.2134/agronj1988.00021962008000060014x

Bangar, K. S., Parmar, B. B., \& Maini, A. (2000). Effect of nitrogen and pressmud cake application on yield and uptake of N, P and K by sugarcane (Saccharum officinarum L.). Crop Research (Hisar), 19, 198-203.

Bennett, H. W. (1959). The effectiveness of selection for the hard seed character in crimson clover. Agronomy Journal, 51, 15-16. http://dx.doi.org/10.2134/agronj1959.00021962005100010006x

Bhatt, R. M., \& Srinivasa Rao, N. K. (2009). Physiology, crop growth, development and yield. In B. S. Dhankhar \& R. Singh (Eds.), Okra Hand Book, Okra Hand Book, Global Production, Processing and Crop Improvement (pp. 37-60). HNB Publishing, New York.

Bhatt, R. M., \& Srinivasa Rao, N. K. (1998). Germination response to fruit position and temperature in okra (Abelmoschus esculenthus (L.). Indian Journal of Horticulture, 55, 81-84.

Cabrera, E. R., Baskin, C. C., \& Nsapato, L. E. (1995). Seed maturation and establishment of hardseededness in Pinkeye Purple hull southernpea in Mississippi. Bulletin 1036. Published by the Office of agricultural Communications, Division of Agriculture, Forestry and Veterinary Medicine, Mississippi State University.

Chaurasia, S. N. S., \& Singh, K. P. (1999). Integration of crop raising methods and organic sources of nutrients on potato production (p. 127). Proceedings of the Global Conference on Potato, 6-11 Dec. New Delhi.

Demir, I. (1997). Occurrence of hardseededness in relation to seed development in okra (Abelmoschus esculentus (L) Moench). Plant Varieties and Seeds, 10, 7-13.

Demir, I. (2001). The effects of heat treatments on hardseedness of serially harvested okra seed lots at optimum and low temperatures. Scientia Horticulturae, 89, 1-7. http://dx.doi.org/10.1016/S0304-4238(00)00216-8

Dutta, O. P., \& Naik, L. B. (2009). Seed production, technology. In B. S. Dhankhar \& R. Singh (Eds.), Okra Hand Book, Global Production, Processing and Crop Improvement (pp. 227-246). HNB Publication, New York.

Duzyaman, E., \& Vural, H. (2009). Managing the variability in okra breeding programs by considering the preferences of the domestic market. Acta Horticulturae, 598, 129-135.

El Balla, M. M. A., Saidahmed, A. I., \& Makkawi, M. (2011). Effect of moisture content and maturity on hardseededness and germination in okra (Abelmoschus esculentus L. Moench). International Journal of Plant Physiology and Biochemistry, 3(6), 102-107. 
FEK. (2000). Greek governmental newspaper.

George, R. A. T. (1987). Technical guidelines of vegetable seed technology. Food and Agriculture Organization of the United Nation, Rome.

ISTA. (2009). International rules for seed testing. Seed Sci. \& Technol. International Seed Testing Association, Switzerland.

Koutsos, T. V. (2009). Greek okra cultivars-sustainable agricultural systems. In B. S. Dhankhar \& R. Singh (Eds.), Okra Hand Book, Global Production, Processing and Crop Improvement (pp. 361-373). New York: HNB Publishing.

Lamont, W. (1999). Okra a versatile vegetable crop. HortTechnology, 9, 179-184.

Majanbu, I. S., Ogunlela, V. B., Ahmed, M. K., \& Olarewaju, J. D. (1985). Response of two okra (Abelmoschus esculentus L. Moench). Varieties to fertilizers: yield and yield components as influenced by nitrogen and phosphorus application. Fertilizer Research, 6, 257-267. http://dx.doi.org/10.1007/BF01048799

Malik, Y. S., Singh, N., Nehra, B. K., Khurana, S. C., Dahiya, M. S., \& Singh, N. (2000). Okra seed quality as influenced by position of pod on mother plant, ripening stage of pod and ambient storage conditions. Haryana Journal of Horticultural Science, 29, 229-232.

Mohammadi, G., Khah, E. M., \& Bannayan, A. M. (2011). Differential responses for harvesting times and storage on hardness of different varieties of okra. Notulae Scientia Biologicae, 3(4), 117-122.

Moniruzzaman, M., \& Quamruzzaman, A. K. M. (2009). Effect of nitrogen levels and picking of green fruits on the fruit and seed production of okra (Abelmoschus esculentus (L.) Moench). Journal of Agriculture and Rural Development, 7, 99-106.

Moore, R. P. (1985). Handbbok on tetrazolium testing (p. 99). I.S.T.A., Zurich.

Naik, L. B., Vogeesha, H. S., Bhanuprakash, K., \& Padmini, K. (2004). Production of open pollinated seeds in okra. Annual Report, 2003-04 (p. 44). Indian Institute of Horticultural Research, Bangalore, India.

Olasantan, F. O. (1994). Fertilizer use in vegetable production in Nigeria. Outlook on Agriculture, 23, 213-222.

Olasantan, F. O. (1999). Nitrogen fertilization of okra (Abelmoschus esculentus) in an intercropping system with cassava (Manihot esculenta) and maize (Zea mays) in south-western Nigeria. Journal of Agricultural Science (Cambridge), 133, 325-334. http://dx.doi.org/10.1017/S0021859699007054

Passam, H. C., \& Polyzou, P. (1997). Improvement of okra seed germination by acid, osmoconditioning and hot water treatments. Plant Varieties and Seeds, 10, 135-140.

Passam, H. C., \& Rekoumi, K. (2009). Okra in Greece. Production and Constraints. In B. S. Dhankhar \& R. Singh (Eds.), Okra Hand Book, Global Production, Processing and Crop Improvement (pp. 347-359). HNB Publishing, N.Y.

Passam, H. C., Theodoropoulou, S., Karanissa, T., \& Karapanos, I. C. (2010). Influence of harvest time and after ripening on the seed quality of eggplant. Scientia Horticulturae, 125, 518-520. http://dx.doi.org/10.1016/j.scienta.2010.04.007

Prabhakar, B. S., Hegde, D. M., Srinivas, K., \& Doijode, S. C. (1985). Seed quality and productivity of okra in relation to nodal position of pod. South Indian Horticulture, 33(2), 115-117.

Prabhakar, M., Hebbar, S. S., \& Gayathri, M. (2009). Production technology. In B. S. Dhankhar \& R. Singh (Eds.), Okra Hand Book, Global Production, Proc-essing and Crop Improvement (pp. 159-174). HNB Publishing, N.Y.

Purquerio, L .F. V., Lago, A. A., \& Passos, F. A. (2010). Germination and hardseedness of seeds in okra elite lines. Horticulturae Brasileira, 28, 232-235. http://dx.doi.org/10.1590/S0102-05362010000200017

Raji, J. A. (1994). Field and glass-house screening of okra (Hibiscus esculentus) genotypes for yield and intercropping. The Indian Journal of Agricultural Sciences, 64, 454-458.

Rekoumi, K., Karapanos, I. C., Akoumianakis, K. A., \& Passam, H. C. (2012). Nitrogen application affects yield and postharvest quality of okra (Hibiscus esculentus L. cv. 'Boyiatiou'). International Journal of Plant Production, 6(1), 59-72.

Rekoumi, K., Moustakas, N., Passam, H., \& Akoumianakis, K. (2013). The effect of nitrogen fertilization on the concentration of inorganic nutrients in okra (Hibiscus esculentus) cv. Boyiatiou. Proceedings of the Greek 
Society of Horticultural Sciences, 10, 424-426.

Shrestha, G. K. (1983). Effect of spacing and nitrogen fertilizer on Pusa Sawani okra A. esculentus in Nepal. Experimental Agriculture, 19(3), 239-242. http://dx.doi.org/10.1017/S0014479700022754

Singh, D. K., Gulshan, L., \& Jain, S. K. (1999). Response of plant bioregulators and foliar feeding of urea on seed yield of okra cv. Pusa sawani during spring summer season. Scientific Horticulture, 6, 121-124.

Singh, R. V. (1995). Effect of nitrogen and phosphorus on performance of rainfed okra (Hibiscus esculentus L.) and cowpea (Vigna unguiculata) intercropping system. Indian Journal of Agronomy, 40, 581-586.

Uddin, M. M., Mondal, M. F., Samsuzzaman, S., \& Siddique, M. A. (2006). Effect of different levels of nitrogen, phosphorous and potassium on the yield and quality of okra seed. In M. N. Uddin, M. A. Rashid \& M. S. Mullah (Eds.), Research Report on Horticultural Crops 2005-2006 (pp. 108-114). Horticulture Research centre, Bangladesh Agricultural Research Institute, Joydebpur, Gazipur.

Wiese, A. M., \& Binning, L. K. (1987). Calculating the threshold temperature of development for weeds. Weed Science, 35, 177-179.

Yadav, S. K., \& Dhankhar, B. S. (1999). Performance of Varsha Uphar cultivar of okra (Abelmoschus esculentus (L.) Moench) as effected by the sowing dates and plant geometry. Vegetable Science, 26, 180-182.

Yadav, S. K., Dhankar, B. S., Deshwal, D. P., \& Tomer, R. P. S. (2001). Effect of sowing date and plant geometry on seed production and quality of okra (Abelmoschus esculenthus (L.) Moench) cv. Varsha Uphar. Seed Research, 29(2), 149-152.

\section{Copyrights}

Copyright for this article is retained by the author(s), with first publication rights granted to the journal.

This is an open-access article distributed under the terms and conditions of the Creative Commons Attribution license (http://creativecommons.org/licenses/by/3.0/). 